\title{
LIMITING ABSORPTION PRINCIPLE AND WELL-POSEDNESS FOR THE TIME-HARMONIC MAXWELL EQUATIONS WITH ANISOTROPIC SIGN-CHANGING COEFFICIENTS
}

\author{
HOAI-MINH NGUYEN AND SWARNENDU SIL
}

\begin{abstract}
We study the limiting absorption principle and the well-posedness of Maxwell equations with anisotropic sign-changing coefficients in the time-harmonic domain. The starting point of the analysis is to obtain Cauchy problems associated with two Maxwell systems using a change of variables. We then derive a priori estimates for these Cauchy problems using two different approaches. The Fourier approach involves the complementing conditions for the Cauchy problems associated with two elliptic equations, which were studied in a general setting by Agmon, Douglis, and Nirenberg. The variational approach explores the variational structure of the Cauchy problems of the Maxwell equations. As a result, we obtain general conditions on the coefficients for which the limiting absorption principle and the well-posedness hold. Moreover, these new conditions are of a local character and easy to check. Our work is motivated by and provides general sufficient criteria for the stability of electromagnetic fields in the context of negative-index metamaterials.
\end{abstract}

Key words: Maxwell equations, sign-changing coefficients, well-posedness, limiting absorption principle, Cauchy problems, resonance, negative-index metamaterials,

AMS subject classifications: 35B34, 35B35, 35B40, 35J05, 78A25.

\section{Contents}

1. Introduction

2. Statement of the main results

3. Fourier approach for the Cauchy problems

3.1. Preliminaries

3.2. Proof of Theorem 2.1

4. Variational approach for the Cauchy problems 14

4.1. Some useful lemmas

4.2. Proof of Theorem 2.2 23

4.3. Some applications of Theorem 2.2

\begin{tabular}{ll|l|}
\hline Appendix A. Proof of Proposition 3.1 & 27 \\
\hline
\end{tabular}

$\begin{array}{ll}\text { References } & 28\end{array}$

\section{INTRODUCTION}

Negative-index metamaterials are artificial structures whose refractive index has a negative value over some frequency range. Their existence was postulated by Veselago in 1964 [36] and confirmed experimentally by Shelby, Smith, and Schultz in 2001 34. Negative-index metamaterial research has been a very active topic of investigation not only because of potentially interesting applications, but also because of challenges involved in understanding 
their peculiar properties due to the sign-changing coefficients in the equations modeling the phenomena.

In this paper, we study the stability of electromagnetic fields in the context of negativeindex metamaterials from a mathematical point of view. More precisely, we study the limiting absorption principle and the well-posedness of Maxwell equations with anisotropic signchanging coefficients in the time-harmonic domain. Let $D$ be an open, bounded subset of $\mathbb{R}^{3}$ of class $C^{1}$ and let $\varepsilon^{+}, \mu^{+}$be defined in $\mathbb{R}^{3} \backslash \bar{D}$, and $\varepsilon^{-}, \mu^{-}$be defined in $D$ such that $\varepsilon^{+}, \mu^{+}$, $-\varepsilon^{-}$, and $-\mu^{-}$are real, symmetric, uniformly elliptic matrix-valued functions. Set, for $\delta \geqslant 0$,

$$
\left(\varepsilon_{\delta}, \mu_{\delta}\right)=\left\{\begin{array}{cl}
\left(\varepsilon^{+}, \mu^{+}\right) & \text {in } \mathbb{R}^{3} \backslash \bar{D}, \\
\left(\varepsilon^{-}+i \delta I, \mu^{-}+i \delta I\right) & \text { in } D .
\end{array}\right.
$$

Here and in what follows, $I$ denotes the $(3 \times 3)$ identity matrix and $\bar{D}$ denotes the closure of $D$. We also denote $B_{R}$ the open ball in $\mathbb{R}^{3}$ centered at the origin and of radius $R>0$. We assume as usual that for some $R_{0}>0, D \subset B_{R_{0}},\left(\varepsilon^{+}, \mu^{+}\right)=(I, I)$ in $\mathbb{R}^{3} \backslash B_{R_{0}}$, and

$$
\varepsilon^{+}, \mu^{+}, \varepsilon^{-}, \mu^{-} \text {are piecewise } C^{1} \text {. }
$$

Given $\delta>0$ and $J \in\left[L^{2}\left(\mathbb{R}^{3}\right)\right]^{3}$ with compact support, let $\left(E_{\delta}, H_{\delta}\right) \in\left[H_{\text {loc }}\left(\operatorname{curl}, \mathbb{R}^{3}\right)\right]^{2}$ be the unique radiating solution of the Maxwell equations

$$
\left\{\begin{array}{cl}
\nabla \times E_{\delta}=i \omega \mu_{\delta} H & \text { in } \mathbb{R}^{3}, \\
\nabla \times H_{\delta}=-i \omega \varepsilon_{\delta} E+J & \text { in } \mathbb{R}^{3} .
\end{array}\right.
$$

Physically, $\varepsilon_{\delta}$ and $\mu_{\delta}$ describe the permittivity and the permeability of the considered medium, $\omega$ is the frequency, $D$ is a plasmonic structure of negative-index metamaterials and $i \delta I$ describes its loss, and $J$ is the density of charge.

The goals of this paper are to derive general conditions on $\left(\varepsilon_{0}, \mu_{0}\right)$ for which $\left(E_{\delta}, H_{\delta}\right)$ are bounded and to characterize its limit. The corresponding scalar version of Maxwell equations in the frequency regime is the Helmholtz equation. The well-posedness of the Helmholtz equation with sign-changing coefficients has been studied intensively, with the initial investigations initiated by Costabel and Stephan [9]. Later, the well-posedness and the Fredholms character were investigated using the integral method [33] and the $T$-coercivity method [5, 7] (and the references therein). Recently, one of the authors [23] obtained general results on the limiting absorbtion principle and the well-posedness for the Helmholtz equation. His approach is based on a priori estimates using Fourier and variational approaches for Cauchy problems associated with two elliptic equations derived naturally in this context. The use of the Cauchy problems in the context of the Helmholtz equations with sign-changing coefficients originally appeared in [19. The theory behind the Helmholtz equations is now almost complete and is the state-of-the-art in this area.

There is very little in the mathematical literature about the stability of the (full) Maxwell equations with sign-changing coefficients. The only known work in this direction that could help us is due to Bonnet-Ben Dhia, Chesnel, and Ciarlet [6] using the $T$-coercivity method. They considered a bounded setting with isotropic coefficients and obtained the well posedness outside a discrete set of $\omega$ under requirements on $\varepsilon$ and $\mu$ of a non-local nature, which is generally difficult to check. The goal of this paper is to fill this gap by developing the approach in 23] for the Maxwell equations. The idea is first to obtain the Cauchy problems associated with two Maxwell systems via a change of variables and then to derive a priori estimates for these Cauchy problems. Two approaches are proposed to obtain these estimates. The Fourier approach involves the complementing conditions for Cauchy problems associated with two elliptic equations, which were studied in more general setting by Agmon, Douglis, and Nirenberg [2]. The variational approach explores the variational structure of the Cauchy 
problems. In comparison with the acoustic setting [23, new ideas are required to handle the complex structure of the Maxwell equations and to be able to apply the theory on complementing conditions for elliptic systems. In particular, various forms of the Poincaré lemma and the Helmholtz decomposition are used with a suitable implementation of local charts to avoid imposing topological conditions. As a result, we obtain very general conditions on the (anisotropic) coefficients to ensure the well-posedness and the limiting absorption principle. Moreover, these new conditions are local and easy to check. For example, the wellposedness and the limiting absorption principle hold under the condition $i)\left(\varepsilon^{+},-\varepsilon^{-}\right)$and $\left(\mu^{+},-\mu^{-}\right)$are smooth near the interface $\partial D$ and both satisfy the complementary condition on the interface (Theorem 2.1 and Corollay 2.1), or $i i) \varepsilon$ and $\mu$ are isotropic near the interface and $\left|\varepsilon^{+}+\varepsilon^{-}\right|$and $\left|\mu^{+}+\mu^{-}\right|$are away from 0 there (Theorem 2.2 and Corollary 4.1), or iii) $\varepsilon^{+},-\varepsilon^{-}, \mu^{+}, \mu^{-}$are equal to $I$ near the interface and the interface is (strictly) convex (Corollary 4.2).

Our work thus provides general sufficient criteria for the stability of electromagnetic fields in the context of negative-index metamaterials in the electromagnetic setting. This work also clarifies the role of the complementary conditions considered in [25] in ensuring the resonance associated with negative-index metamaterials appeared in their various applications, such as superlensing [25] and cloaking [28, 26], in the electromagnetic setting. Previous mathematical works on applications of negative-index metamaterials, such as superlensing, cloaking using complementary media, cloaking via anomalous localized resonance for a source or for an object, can be found in [20], [22, 29], [3, 14, 15, 17, 21, 27, 28, 32], and [24, respectively, and the references therein.

\section{Statement of the main Results}

The starting point of our analysis is to obtain Cauchy problems associated with two Maxwell systems using a change of variables. A central point of the analysis is then to derive a priori estimates for these Cauchy problems. We first use a Fourier approach involving the theory of complementing conditions as developed by Agmon, Douglis, and Nirenberg [2]. Given a unit vector $e \in \mathbb{R}^{3}$, denote

$$
\mathbb{R}_{e,+}^{3}=\left\{x \in \mathbb{R}^{3} ;\langle x, e\rangle>0\right\} \quad \text { and } \quad \mathbb{R}_{e, 0}^{3}=\left\{x \in \mathbb{R}^{3} ;\langle x, e\rangle=0\right\} .
$$

Here and in what follows, $\langle\cdot, \cdot\rangle$ denotes the standard scalar product in $\mathbb{R}^{3}$ or $\mathbb{C}^{3}$. The definition of the complementing condition for the Cauchy problem of two elliptic equations is as follows

Definition 2.1 (Agmon, Douglis, Nirenberg [2]). Two constant, positive, symmetric matrices $A_{1}$ and $A_{2}$ are said to satisfy the (Cauchy) complementing boundary condition with respect to direction $e \in \partial B_{1}$ if and only if for all $\xi \in \mathbb{R}_{e, 0}^{3} \backslash\{0\}$, the only solution $\left(u_{1}(x), u_{2}(x)\right)$ of the form $\left(e^{i\langle y, \xi\rangle} v_{1}(t), e^{i\langle y, \xi\rangle} v_{2}(t)\right)$ with $x=y+$ te where $t=\langle x, e\rangle$, of the following system

$$
\left\{\begin{array}{c}
\operatorname{div}\left(A_{1} \nabla u_{1}\right)=\operatorname{div}\left(A_{2} \nabla u_{2}\right)=0 \text { in } \mathbb{R}_{e,+}^{3}, \\
u_{1}=u_{2} \text { and } A_{1} \nabla u_{1} \cdot e=A_{2} \nabla u_{2} \cdot e \text { on } \mathbb{R}_{e, 0}^{3},
\end{array}\right.
$$

that is bounded in $\mathbb{R}_{e,+}^{3}$ is $(0,0)$.

Remark 2.1. Agmon, Douglis, and Nirenberg [2] (see also [1]) considered the complementing conditions for a general elliptic system and derived properties for them. The special case given in the above definition found a particular interest in the context of the Helmholtz equations with sign-changing coefficients (see [23]).

Denote

$$
\Gamma=\partial D
$$


and, for $\tau>0$, set

$$
D_{\tau}=\{x \in D ; \operatorname{dist}(x, \Gamma)<\tau\} \quad \text { and } \quad D_{-\tau}=\left\{x \in \mathbb{R}^{3} \backslash \bar{D} ; \operatorname{dist}(x, \Gamma)<\tau\right\} .
$$

For the Fourier approach, we prove the following result:

Theorem 2.1. Let $0<\delta<1, J \in\left[L^{2}\left(\mathbb{R}^{3}\right)\right]^{3}$ with supp $J \subset B_{R_{0}}$, and let $\left(E_{\delta}, H_{\delta}\right) \in$ $\left[H_{\text {loc }}\left(\text { curl, } \mathbb{R}^{3}\right)\right]^{2}$ be the unique radiating solution of $(1.3)$. Assume that $\varepsilon^{+}, \mu^{+} \in C^{1}\left(\bar{D}_{-\tau}\right)$, $\varepsilon^{-}, \mu^{-} \in C^{1}\left(\bar{D}_{\tau}\right)$ for some $\tau>0$, and $\left(\varepsilon^{+},-\varepsilon^{-}\right)(x)$ and $\left(\mu^{+},-\mu^{-}\right)(x)$ satisfy the (Cauchy) complementing conditions with respect to the unit normal vector $\nu(x)$ for all $x \in \Gamma$. Then

$$
\left\|\left(E_{\delta}, H_{\delta}\right)\right\|_{L^{2}\left(B_{R}\right)} \leqslant C_{R}\|J\|_{L^{2}\left(\mathbb{R}^{3}\right)} \quad \forall R>0,
$$

for some positive constant $C_{R}$ independent of $\delta$ and $J$. Moreover, $\left(E_{\delta}, H_{\delta}\right)$ converges to $\left(E_{0}, H_{0}\right)$ strongly in $\left[L_{\mathrm{loc}}^{2}\left(\mathbb{R}^{3}\right)\right]^{6}$ as $\delta \rightarrow 0$, where $\left(E_{0}, H_{0}\right) \in\left[H_{\mathrm{loc}}\left(\mathrm{curl}, \mathbb{R}^{3}\right)\right]^{2}$ is the unique radiating solution of (1.3) with $\delta=0$. As a consequence, we have

$$
\left\|\left(E_{0}, H_{0}\right)\right\|_{L^{2}\left(B_{R}\right)} \leqslant C_{R}\|J\|_{L^{2}\left(\mathbb{R}^{3}\right)} \quad \forall R>0 .
$$

Let $\Omega$ be an open set of $\mathbb{R}^{3}$. Given $\left(u_{n}\right) \subset L_{\text {loc }}^{2}(\Omega)$ and $u \in L_{\text {loc }}^{2}(\Omega)$, one says that $u_{n}$ converges to $u$ in $L_{\text {loc }}^{2}(\Omega)$ if $u_{n}$ converges to $u$ in $L^{2}(K)$ for every compact subset $K$ of $\Omega$. Recall that, for $\omega>0$, a solution $(E, H) \in\left[H_{\text {loc }}\left(\operatorname{curl}, \mathbb{R}^{3} \backslash B_{R}\right)\right]^{2}$, for some $R>0$, of the Maxwell equations

$$
\begin{cases}\nabla \times E=i \omega H & \text { in } \mathbb{R}^{3} \backslash B_{R}, \\ \nabla \times H=-i \omega E & \text { in } \mathbb{R}^{3} \backslash B_{R},\end{cases}
$$

is called radiating if it satisfies one of the (Silver-Müller) radiation conditions

$$
H \times x-|x| E=O(1 /|x|) \quad \text { or } \quad E \times x+|x| H=O(1 /|x|) \quad \text { as }|x| \rightarrow+\infty .
$$

Here and in what follows, for $\alpha \in \mathbb{R}, O\left(|x|^{\alpha}\right)$ denotes a quantity whose norm is bounded by $C|x|^{\alpha}$ for some constant $C>0$. One also denotes

$$
\begin{gathered}
H(\operatorname{curl}, \Omega)=\left\{u \in\left[L^{2}(\Omega)\right]^{3} ; \nabla \times u \in\left[L^{2}(\Omega)\right]^{3}\right\}, \\
H_{\mathrm{loc}}(\operatorname{curl}, \Omega)=\left\{u \in\left[L_{\mathrm{loc}}^{2}(\Omega)\right]^{3} ; \nabla \times u \in\left[L_{\mathrm{loc}}^{2}(\Omega)\right]^{3}\right\}, \\
H(\operatorname{div}, \Omega)=\left\{u \in\left[L^{2}(\Omega)\right]^{3} ; \operatorname{div} u \in L^{2}(\Omega)\right\},
\end{gathered}
$$

and, on $\partial \Omega, \nu$ the unit normal vector directed to the exterior of $\Omega$.

The proof of Theorem 2.1 is given in Section 3. The existence and uniqueness of $\left(E_{0}, H_{0}\right)$ are also established there. The analysis given in Section 3 requires a number of tools and results, such as Poincaré's lemma, the Helmholtz decomposition for both electric and magnetic fields, the analysis on the complementing conditions, duality arguments, and a result on the trace estimate for $H(\operatorname{div}, \Omega)$ (Lemma 3.4), which is interesting in its own right. From the analysis, we derive that the complementing conditions for the Cauchy problem associated with two Maxwell systems follows from the complementing conditions for the Cauchy problems associated with two elliptic equations corresponding to the pair of permittivity and permeability. To the best of our knowledge, this insight is new, and its discovery makes the required conditions on $\varepsilon$ and $\mu$ easy to check (see Proposition 2.1 below). The optimality of the complementing conditions is discussed in Proposition 3.1, whose proof is given in the appendix.

To check the complementing condition, one can use its following algebraic characterization (see [23, Proposition 1]). 
Proposition 2.1. Let $e \in \partial B_{1}$, and let $A_{1}$ and $A_{2}$ be two constant, positive, symmetric matrices. Then $A_{1}$ and $A_{2}$ satisfy the (Cauchy) complementing condition with respect to $e$ if and only if

$$
\left\langle A_{2} e, e\right\rangle\left\langle A_{2} \xi, \xi\right\rangle-\left\langle A_{2} e, \xi\right\rangle^{2} \neq\left\langle A_{1} e, e\right\rangle\left\langle A_{1} \xi, \xi\right\rangle-\left\langle A_{1} e, \xi\right\rangle^{2} \quad \forall \xi \in \mathbb{R}_{e, 0}^{3} \backslash\{0\} .
$$

In particular, if $A_{2}>A_{1}$, then $A_{1}$ and $A_{2}$ satisfy the complementing condition with respect to $e$.

In this paper, for two $(3 \times 3)$ matrices $A$ and $B$, the notation $A>B$ means that $\langle A x, x\rangle>$ $\langle B x, x\rangle$ for all $x \in \mathbb{R}^{3} \backslash\{0\}$. A similar convention is used for $A \geqslant B$. As a direct consequence of Theorem 2.1 and Proposition 2.1, one obtains

Corollary 2.1. Let $0<\delta<1, J \in\left[L^{2}\left(\mathbb{R}^{3}\right)\right]^{3}$ with supp $J \subset B_{R_{0}}$, and let $\left(E_{\delta}, H_{\delta}\right) \in$ $\left[H_{\mathrm{loc}}\left(\mathrm{curl}, \mathbb{R}^{3}\right)\right]^{2}$ be the unique radiating solution of $(1.3)$. Assume that $\varepsilon^{+}, \mu^{+} \in C^{1}\left(\bar{D}_{-\tau}\right)$ and $\varepsilon^{-}, \mu^{-} \in C^{1}\left(\bar{D}_{\tau}\right)$ for some $\tau>0$, and for each connected component of $\Gamma$,

$$
\left(\varepsilon^{+} \geqslant-\varepsilon^{-}+c I \quad \text { or } \quad-\varepsilon^{-} \geqslant \varepsilon^{+}+c I\right),
$$

and

$$
\left(\mu^{+} \geqslant-\mu^{-}+c I \quad \text { or } \quad-\mu^{-} \geqslant \mu^{+}+c I\right),
$$

for some $c>0$. Then

$$
\left\|\left(E_{\delta}, H_{\delta}\right)\right\|_{L^{2}\left(B_{R}\right)} \leqslant C_{R}\|J\|_{L^{2}\left(\mathbb{R}^{3}\right)} \quad \forall R>0,
$$

for some positive constant $C_{R}$ independent of $\delta$ and $J$. Moreover, $\left(E_{\delta}, H_{\delta}\right)$ converges to $\left(E_{0}, H_{0}\right)$ strongly in $\left[L_{\mathrm{loc}}^{2}\left(\mathbb{R}^{3}\right)\right]^{6}$ as $\delta \rightarrow 0$, where $\left(E_{0}, H_{0}\right) \in\left[H_{\mathrm{loc}}\left(\mathrm{curl}, \mathbb{R}^{3}\right)\right]^{2}$ is the unique radiating solution of (1.3) with $\delta=0$. As a consequence, we have

$$
\left\|\left(E_{0}, H_{0}\right)\right\|_{L^{2}\left(B_{R}\right)} \leqslant C_{R}\|J\|_{L^{2}\left(\mathbb{R}^{3}\right)} \quad \forall R>0 .
$$

Theorem 2.1 (see also its consequence Corollary 2.1) provides very general conditions for ensuring the well-posedness and the validity of the limiting absorption principle. One does not require that $\varepsilon^{+}+\varepsilon^{-}$and $\mu^{+}+\mu^{-}$have the same sign on $\Gamma$. This was previously out of reach.

Our next approach for addressing the Cauchy problems is a variational one. To this end, we first introduce some notations.

Definition 2.2. Let $\tau>0$ and $U$ be a smooth, open subset of $\mathbb{R}^{3}$ such that $\bar{U} \subset D$. $A$ transformation $\mathcal{F}: D \backslash \bar{U} \rightarrow D_{-\tau}$ is said to be a reflection through $\Gamma$ if and only if $\mathcal{F}$ is a diffeomorphism and $\mathcal{F}(x)=x$ on $\Gamma$.

Here and in what follows, when we mention a diffeomorphism $\mathcal{F}: \Omega \rightarrow \Omega^{\prime}$ for two open subsets $\Omega$ and $\Omega^{\prime}$ of $\mathbb{R}^{3}$, we mean that $\mathcal{F}$ is a diffeomorphism, $\mathcal{F} \in C^{1}(\bar{\Omega})$, and $\mathcal{F}^{-1} \in C^{1}\left(\bar{\Omega}^{\prime}\right)$. For a diffeomorphism $\mathcal{F}$ from $\Omega$ to $\Omega^{\prime}$, for a matrix $A$ defined in $\Omega$, and for a vector field $\mathcal{E}$ defined in $\Omega$, denote

$$
\mathcal{F}_{*} A\left(x^{\prime}\right)=\frac{\nabla \mathcal{F}(x) A(x) \nabla \mathcal{F}^{T}(x)}{\mathcal{J}(x)} \quad \text { and } \quad \mathcal{F} * \mathcal{E}\left(x^{\prime}\right)=\nabla \mathcal{F}^{-T}(x) \mathcal{E}(x),
$$

with $x=\mathcal{F}^{-1}\left(x^{\prime}\right)$ and $\mathcal{J}(x)=\operatorname{det} \nabla \mathcal{F}(x)$. For a vector field $J$ defined in $\Omega$, we set

$$
\mathcal{T}_{*} J\left(x^{\prime}\right)=\frac{J(x)}{\mathcal{J}(x)} .
$$


In what follows, $d_{\partial \Omega}$ denotes the distance function to $\partial \Omega$ for an open, bounded subset $\Omega$ of $\mathbb{R}^{3}$, i.e.,

$$
d_{\partial \Omega}(x):=\min \{|y-x| ; y \in \partial \Omega\} \text { for } x \in \mathbb{R}^{3} .
$$

The main result in this direction is

Theorem 2.2. Let $0<\delta<1, \tau>0, J \in\left[L^{2}\left(\mathbb{R}^{3}\right)\right]^{3}$ with supp $J \subset B_{R_{0}}$, and let $\left(E_{\delta}, H_{\delta}\right) \in$ $\left[H_{\mathrm{loc}}\left(\mathrm{curl}, \mathbb{R}^{3}\right)\right]^{2}$ be the unique radiating solution of $(1.3)$. Assume that there exist a smooth open subset $U$ of $\mathbb{R}^{d}$ with $\bar{U} \subset D$ and a reflection $\mathcal{F}$ through $\Gamma$ from $D \backslash \bar{U}$ onto $D_{-\tau}$ such that, with the notations

$$
(\varepsilon, \mu)=\left(\varepsilon^{+}, \mu^{+}\right) \text {in } D_{-\tau} \quad \text { and } \quad(\hat{\varepsilon}, \hat{\mu})=\left(\mathcal{F}_{*} \varepsilon^{-}, \mathcal{F}_{*} \mu^{-}\right) \text {in } D_{-\tau},
$$

for each connected component $O$ of $D_{-\tau}$,

$$
\left(\hat{\varepsilon}-\varepsilon \geqslant c d_{\Gamma}^{\alpha_{1}} I \text { in } O \quad \text { or } \quad \varepsilon-\hat{\varepsilon} \geqslant c d_{\Gamma}^{\alpha_{1}} I \text { in } O\right)
$$

and

$$
\left(\hat{\mu}-\mu \geqslant c d_{\Gamma}^{\alpha_{2}} I \text { in } O \quad \text { or } \quad \mu-\hat{\mu} \geqslant c d_{\Gamma}^{\alpha_{2}} I \text { in } O\right),
$$

for some $0 \leqslant \alpha_{1}, \alpha_{2}<2$ and for some positive constant $c$. In the case $\alpha_{1}+\alpha_{2}>0$ in $O$, additionally assume that

$$
\text { supp } J \cap\left(O \cup \mathcal{F}^{-1}(O)\right)=\varnothing .
$$

Set, in $D_{-\tau}$,

$$
\hat{E}_{\delta}=\mathcal{F} * E_{\delta} \quad \text { and } \quad \hat{H}_{\delta}=\mathcal{F} * H_{\delta}
$$

and additionally assume that $D$ is of class $C^{2}$. Then, for all $R>0$ and for all open set $V$ containing $\Gamma$,

$$
\begin{aligned}
\int_{B_{R} \backslash V}\left|\left(E_{\delta}, H_{\delta}\right)\right|^{2}+\int_{D_{-\tau}}\left|\left\langle(\varepsilon-\hat{\varepsilon}) E_{\delta}, E_{\delta}\right\rangle\right| & +\left|\left\langle(\mu-\hat{\mu}) H_{\delta}, H_{\delta}\right\rangle\right| \\
& +\int_{D_{-\tau}}\left|\left(E_{\delta}-\hat{E}_{\delta}, H_{\delta}-\hat{H}_{\delta}\right)\right|^{2} \leqslant C\|J\|_{L^{2}\left(\mathbb{R}^{3}\right)}^{2},
\end{aligned}
$$

for some positive constant $C=C_{R, V}$ independent of $\delta$ and $J$. Moreover, for any sequence $\left(\delta_{n}\right)$ converging to 0 , up to a subsequence, $\left(E_{\delta_{n}}, H_{\delta_{n}}\right)$ converges to $\left(E_{0}, H_{0}\right)$ strongly in $\left[L_{\text {loc }}^{2}\left(\mathbb{R}^{3} \backslash \Gamma\right)\right]^{6}$ as $n \rightarrow+\infty$, where $\left(E_{0}, H_{0}\right) \in\left[H_{\mathrm{loc}}\left(\operatorname{curl}, \mathbb{R}^{3} \backslash \Gamma\right)\right]^{2}$ satisfies (2.3) with $\delta=0$ and is a radiating solution of (1.3) with $\delta=0$. In the case $\alpha_{1}=\alpha_{2}=0$ for all connected components of $D_{-\tau}$, the convergence holds in $\left[L_{\mathrm{loc}}^{2}\left(\mathbb{R}^{3}\right)\right]^{6}$ for $\delta \rightarrow 0$, and the limit $\left(E_{0}, H_{0}\right) \in\left[H_{\text {loc }}\left(\text { curl, } \mathbb{R}^{3}\right)\right]^{2}$ is unique.

The meaning of the radiating solution of $\left(E_{0}, H_{0}\right)$ in Theorem 2.2 is understood as follows. A pair $\left(E_{0}, H_{0}\right) \in\left[H_{\text {loc }}\left(\operatorname{curl}, \mathbb{R}^{3} \backslash \Gamma\right)\right]^{2}$ satisfies (2.3) with $\delta=0$ and is called a radiating solution of (1.3) with $\delta=0$ if $\left(E_{0}, H_{0}\right)$ is a radiating solution of

$$
\left\{\begin{array}{cc}
\nabla \times E_{0}=i \omega \mu_{0} H & \text { in } \mathbb{R}^{3} \backslash \Gamma, \\
\nabla \times H_{0}=-i \omega \varepsilon_{0} E+J & \text { in } \mathbb{R}^{3} \backslash \Gamma,
\end{array}\right.
$$

and it satisfies

$$
\left(E_{0}-\hat{E}_{0}\right) \times \nu=0=\left(H_{0}-\hat{H}_{0}\right) \times \nu \text { on } \Gamma \text {. }
$$

Note that (2.4) makes sense since, if $\alpha_{1}+\alpha_{2}>0$ in $O$,

$$
\nabla\left(E_{0}-\hat{E}_{0}\right)=i \omega \mu H_{0}-i \omega \hat{\mu} \hat{H}_{0}=i \omega \mu\left(H_{0}-\hat{H}_{0}\right)+i \omega(\mu-\hat{\mu}) \hat{H}_{0} \text { in } O
$$

and

$$
\nabla\left(H_{0}-\hat{H}_{0}\right)=i \omega \varepsilon E_{0}-i \omega \hat{\varepsilon} \hat{E}_{0}=i \omega \varepsilon\left(E_{0}-\hat{E}_{0}\right)+i \omega(\varepsilon-\hat{\varepsilon}) \hat{E}_{0} \text { in } O
$$


One can then check, in both cases $\alpha_{1}+\alpha_{2}>0$ in $O$ or $\alpha_{1}+\alpha_{2}=0$ in $O$, that

$$
E_{0}-\hat{E}_{0} \in H\left(\operatorname{curl}, D_{-\tau}\right) \quad \text { and } \quad H_{0}-\hat{H}_{0} \in H\left(\operatorname{curl}, D_{-\tau}\right)
$$

using (2.3) with $\delta=0$.

The proof of Theorem 2.2 is given in Section 4. The variational structure is explored in Lemma 4.5. Important ingredients in the proof are two forms of Poincaré's lemmas: one with a gain in the integrability (Lemmas 4.1 and 4.2) and one with a gain of regularity (Lemma 4.4). We also suitably use local charts to avoid imposing topological conditions. Nevertheless, a more global approach in comparison with the proof of Theorem 2.1 is required in order to remove undesirable terms in the process of using local charts (see Remark 4.2). Some applications of Theorem 2.2 are given in Section 4.3 .

Let $B_{r}(x)$ denote the open ball in $\mathbb{R}^{3}$ centered at $x$ and of radius $r$ for $x \in \mathbb{R}^{3}$ and for $r>0$. It is shown in [28, Proposition 2.2] that if $\hat{\varepsilon}=\varepsilon$ and $\hat{\mu}=\mu$ in $B_{r}\left(x_{0}\right) \cap D_{-\tau}$ for some $x_{0} \in \Gamma$ and $r>0$, then resonance may occur. These requirements on $\varepsilon, \mu, \hat{\varepsilon}$, and $\hat{\mu}$ are related to the complementary property of media [25, Definition 1]. This property plays a role in the construction of lensing and cloaking devices using negative-index materials [25, 26] for which a localized resonance can take place, an interesting phenomenon of negative-index metamaterials in which the solutions blow up in some regions and remain bounded in others as the loss goes to 0 .

The rest of the paper is organized as follows. The proof of Theorems 2.1 and 2.2 are given in Sections 3 and 4, repectively. In the appendix, we present the proof of Proposition 3.1 on the optimality of complementing conditions for the Cauchy problem associated with two Maxwell systems.

\section{Fourier approach for the CAUChy problems}

This section is devoted to the proof of Theorem 2.1. We first establish several lemmas in Section 3.1 which are used in the proof of Theorem 2.1. The proof of Theorem 2.1 is given in Section 3.2. The optimality of the complementing conditions used in Theorem 2.1 is discussed in Proposition 3.1 whose proof is given in the appendix.

3.1. Preliminaries. We begin this section by recall the following known form of Poincaré's lemma, see e.g. [12, Theorem 3.4].

Lemma 3.1. Let $\Omega$ be a simply connected, bounded, open subset of $\mathbb{R}^{3}$ of class $C^{1}$. There exists a linear, continuous transformation $T: H(\operatorname{curl}, \Omega) \rightarrow\left[H^{1}(\Omega)\right]^{3}$ such that

$$
\nabla \times T(u)=\nabla \times u \text { in } \Omega .
$$

We next establish the key ingredient of the proof of Theorem 2.1.

Lemma 3.2. Let $\Omega$ be a simply connected, bounded, open subset of $\mathbb{R}^{3}$ of class $C^{1}$. Let $\varepsilon, \hat{\varepsilon}, \mu, \hat{\mu}$ be real, symmetric, uniformly elliptic matrix-valued functions defined in $\Omega$ and of class $C^{1}$. Let $J_{e}, J_{m}, \hat{J}_{e}, \hat{J}_{m} \in\left[L^{2}(\Omega)\right]^{3}$ and let $(E, H),(\hat{E}, \hat{H}) \in[H(\operatorname{curl}, \Omega)]^{2}$ be such that

$$
\left\{\begin{array} { c l } 
{ \nabla \times E = i \omega \mu H + J _ { e } } & { \text { in } \Omega , } \\
{ \nabla \times H = - i \omega \varepsilon E + J _ { m } } & { \text { in } \Omega , }
\end{array} \quad \left\{\begin{array}{cl}
\nabla \times \hat{E}=i \omega \hat{\mu} \hat{H}+\hat{J}_{e} & \text { in } \Omega, \\
\nabla \times \hat{H}=-i \omega \hat{\varepsilon} \hat{E}+\hat{J}_{m} & \text { in } \Omega,
\end{array}\right.\right.
$$

and

$$
E \times \nu=\hat{E} \times \nu \quad \text { and } \quad H \times \nu=\hat{H} \times \nu \text { on } \partial \Omega
$$

Set

$$
\mathbf{E}=T(E), \quad \hat{\mathbf{E}}=T(\hat{E}), \quad \mathbf{H}=T(H), \quad \hat{\mathbf{H}}=T(\hat{H}) \quad \text { in } \Omega,
$$

where $T$ is the operator given in Lemma 3.1. We have 
1) If $(\varepsilon, \hat{\varepsilon})$ satisfies the complementing condition with respect to $\nu(x)$ for all $x \in \partial \Omega$, then

$$
\begin{aligned}
& \|(E-\mathbf{E}, \hat{E}-\hat{\mathbf{E}})\|_{L^{2}(\Omega)} \\
& \quad \leqslant C\left(\left\|\left(\mathbf{E}, \hat{\mathbf{E}}, J_{m}, \hat{J}_{m}\right)\right\|_{L^{2}(\Omega)}+\|\mathbf{E}-\hat{\mathbf{E}}\|_{H^{-1 / 2}(\partial \Omega)}+\|(E-\mathbf{E}, \hat{E}-\hat{\mathbf{E}})\|_{\left[H^{1}(\Omega)\right]^{*}}\right) .
\end{aligned}
$$

2) If $(\mu, \hat{\mu})$ satisfies the complementing condition with respect to $\nu(x)$ for all $x \in \partial \Omega$, then

$$
\begin{aligned}
& \|(H-\mathbf{H}, \hat{H}-\hat{\mathbf{H}})\|_{L^{2}(\Omega)} \\
& \quad \leqslant C\left(\left\|\left(\mathbf{H}, \hat{\mathbf{H}}, J_{e}, \hat{J}_{e}\right)\right\|_{L^{2}(\Omega)}+\|\mathbf{H}-\hat{\mathbf{H}}\|_{H^{-1 / 2}(\partial \Omega)}+\|(H-\mathbf{H}, \hat{H}-\hat{\mathbf{H}})\|_{\left[H^{1}(\Omega)\right]^{*}}\right) .
\end{aligned}
$$

Here $C$ denotes a positive constant depending only on $\varepsilon, \mu$, and $\Omega$.

Here and in what follows, $\left[H^{1}(\Omega)\right]^{*}$ denotes the dual space of $H^{1}(\Omega)$.

Proof. We only prove assertion 1). Assertion 2) can be obtained similarly and its proof is omitted. From the properties of $T$, we have

$$
\nabla \times(E-\mathbf{E})=\nabla \times(\hat{E}-\hat{\mathbf{E}})=0 \quad \text { in } \Omega .
$$

Since $\Omega$ is simply connected, there exists $\varphi, \hat{\varphi} \in H^{1}(\Omega)$ such that

$$
E-\mathbf{E}=\nabla \varphi, \quad \hat{E}-\hat{\mathbf{E}}=\nabla \hat{\varphi} \quad \text { in } \Omega, \quad \text { and } \quad \int_{\Omega} \varphi=\int_{\Omega} \hat{\varphi}=0 .
$$

We have, by the second equation of the system of $(E, H)$,

$$
\operatorname{div}(\varepsilon \nabla \varphi)=\operatorname{div}(\varepsilon E)-\operatorname{div}(\varepsilon \mathbf{E})=-\operatorname{div}(\varepsilon \mathbf{E})-\frac{i}{\omega} \operatorname{div}\left(J_{m}\right) \text { in } \Omega,
$$

by the second equation of the system of $(\hat{E}, \hat{H})$,

$$
\begin{gathered}
\operatorname{div}(\hat{\varepsilon} \nabla \hat{\varphi})=\operatorname{div}(\hat{\varepsilon} \hat{E})-\operatorname{div}(\hat{\varepsilon} \hat{\mathbf{E}})=-\operatorname{div}(\hat{\varepsilon} \hat{\mathbf{E}})-\frac{i}{\omega} \operatorname{div}\left(\hat{J}_{m}\right) \text { in } \Omega, \\
(\nabla \varphi-\nabla \hat{\varphi}) \times \nu=(E-\mathbf{E}) \times \nu-(\hat{E}-\hat{\mathbf{E}}) \times \nu=(\hat{\mathbf{E}}-\mathbf{E}) \times \nu \text { on } \partial \Omega,
\end{gathered}
$$

and, on $\partial \Omega$,

$$
\begin{aligned}
&\left(\varepsilon \nabla \varphi+\varepsilon \mathbf{E}+\frac{i}{\omega} J_{m}\right) \cdot \nu-\left(\hat{\varepsilon} \nabla \hat{\varphi}+\hat{\varepsilon} \hat{\mathbf{E}}+\frac{i}{\omega} \hat{J}_{m}\right) \cdot \nu \\
&=\left(\varepsilon E+\frac{i}{\omega} J_{m}\right) \cdot \nu-\left(\hat{\varepsilon} \hat{E}+\frac{i}{\omega} \hat{J}_{m}\right) \cdot \nu \\
& \quad=-\frac{1}{i \omega} \nabla \times(H-\hat{H}) \cdot \nu=\frac{1}{i \omega} \operatorname{div}_{\Gamma}[(H-\hat{H}) \times \nu]=0 .
\end{aligned}
$$

Since $(\varepsilon, \hat{\varepsilon})$ satisfies the complementing condition, applying [30, Lemma 18] (with $\lambda=1$, $\Sigma_{1}=1, \Sigma_{2}>0$ sufficiently large) and using the standard arguments of freezing coefficients, we derive from (3.4), (3.5), (3.6), and (3.7) that

$$
\|(\nabla \varphi, \nabla \hat{\varphi})\|_{L^{2}(\Omega)} \leqslant C\left(\left\|\left(\mathbf{E}, \hat{\mathbf{E}}, J_{m}, \hat{J}_{m}\right)\right\|_{L^{2}(\Omega)}+\|\mathbf{E}-\hat{\mathbf{E}}\|_{H^{-1 / 2}(\partial \Omega)}+\|(\varphi, \hat{\varphi})\|_{L^{2}(\Omega)}\right) .
$$

We claim that, for $u \in H^{1}(\Omega)$ with $\int_{\Omega} u=0$,

$$
\|u\|_{L^{2}(\Omega)} \leqslant C\|\nabla u\|_{\left[H^{1}(\Omega)\right]^{*}} .
$$


In fact, fix $\xi \in L^{2}(\Omega)$ arbitrary and set $\xi_{\Omega}:=\frac{1}{|\Omega|} \int_{\Omega} \xi$. Let $\varphi \in H^{1}(\Omega)$ be the unique solution of $-\Delta \varphi=\xi-\xi_{\Omega}$ in $\Omega$ and $\partial_{\nu} \varphi=0$ on $\partial \Omega$ with $\int_{\Omega} \varphi=0$. We have

$$
\|\nabla \varphi\|_{L^{2}(\Omega)} \leqslant C\|\xi\|_{L^{2}(\Omega)}
$$

and

$$
\left|\int_{\Omega} u \xi\right|=\left|\int_{\Omega} u\left(\xi-\xi_{\Omega}\right)\right|=\left|\int_{\Omega} \nabla u \nabla \varphi\right| \leqslant\|\nabla u\|_{L^{2}(\Omega)}\|\nabla \varphi\|_{L^{2}(\Omega)}
$$

and the claim follows.

Applying the claim, we have

$$
\|(\varphi, \hat{\varphi})\|_{L^{2}(\Omega)} \leqslant C\|(\nabla \varphi, \nabla \hat{\varphi})\|_{\left[H^{1}(\Omega)\right]^{*}}
$$

and assertion 1) follows from (3.8) and the choice of $\varphi$ and $\hat{\varphi}$ in (3.3).

Remark 3.1. In the proof of Lemma 3.2, we used [30, Lemma 18]. This lemma is in the spirit of the results given in [2] due to Agmon, Douglis, and Nirenberg where more regular data are used.

We next establish a compactness result used in Lemma 3.5 which is a key ingredient of the proof of Theorem 2.1 ,

Lemma 3.3. Let $d \geqslant 2$, and let $O \subset \mathbb{R}^{d}$ be open, bounded, and of class $C^{1}$. Let $\left(u_{n}\right)$ be a bounded sequence in $L^{2}(O)$. Then, up to a subsequence, $\left(u_{n}\right)$ converges in $\left[H^{1}(O)\right]^{*}$.

Proof. Without loss of generality, one may assume that $\left(u_{n}\right)$ converges weakly to $u$ in $L^{2}(O)$. It suffices to prove that, up to a subsequence, $\left(v_{n}\right)$ converges to 0 in $\left[H^{1}(O)\right]^{*}$ where $v_{n}:=$ $u_{n}-u$ in $O$. Let $\varphi_{n} \in H_{0}^{1}(O)$ be the unique solution of $-\Delta \varphi_{n}=v_{n}$ in $O$. Since $\left(v_{n}\right)$ is bounded in $L^{2}(O)$, it follows that $\left(\varphi_{n}\right)$ is bounded in $H^{1}(O)$. Without loss of generality, one may assume that $\left(\varphi_{n}\right)$ converges in $L^{2}(O)$. Then

$$
\int_{O}\left|\nabla \varphi_{n}\right|^{2}=\int_{O} v_{n} \varphi_{n} \rightarrow 0 \text { as } n \rightarrow+\infty
$$

We have, for $\xi \in H^{1}(O)$,

$$
\int_{O} v_{n} \xi=-\int_{O} \Delta \varphi_{n} \xi=\int_{O} \nabla \varphi_{n} \nabla \xi-\int_{\partial O} \partial_{\nu} \varphi_{n} \xi
$$

It follows that

$$
\left\|v_{n}\right\|_{\left[H^{1}(O)\right]^{*}} \leqslant C\left(\left\|\nabla \varphi_{n}\right\|_{L^{2}(O)}+\left\|\partial_{\nu} \varphi_{n}\right\|_{H^{-1 / 2}(\partial O)}\right) .
$$

Here and in what follows in the proof, $C$ denotes a positive constant depending only on $O$. Applying Lemma 3.4 below, we have

$$
\left\|\partial_{\nu} \varphi_{n}\right\|_{H^{-1 / 2}(\partial O)}^{2} \leqslant C\left\|\nabla \varphi_{n}\right\|_{L^{2}(O)}\left(\left\|\nabla \varphi_{n}\right\|_{L^{2}(O)}+\left\|v_{n}\right\|_{L^{2}(O)}\right) \stackrel{(3.9)}{\rightarrow} 0 \text { as } n \rightarrow+\infty \text {. }
$$

The conclusion now follows from (3.9) and (3.10).

In the proof of Lemma 3.3, we use the following result which is interesting in itself.

Lemma 3.4. Let $d \geqslant 2$, and let $O \subset \mathbb{R}^{d}$ be open, bounded, and of class $C^{1}$. Let $u \in H(\operatorname{div}, O)$. We have

$$
\|u \cdot \nu\|_{H^{-1 / 2}(\partial O)}^{2} \leqslant C\|u\|_{L^{2}(O)}\left(\|u\|_{L^{2}(O)}+\|\operatorname{div} u\|_{L^{2}(O)}\right),
$$

for some positive constant $C$ depending only on $O$. 
Remark 3.2. Lemma 3.4 is in the spirit of [13, Lemma A.1]. Estimate (3.11) is stronger than the standard one which asserts that

$$
\|u \cdot \nu\|_{H^{-1 / 2}(\partial O)} \leqslant C\left(\|u\|_{L^{2}(O)}+\|\operatorname{div} u\|_{L^{2}(O)}\right) .
$$

Proof. Using a density argument, one may assume that $u$ is smooth. Using local charts, it suffices to prove that

$$
\|u \cdot \nu\|_{H^{-1 / 2}\left(\partial \mathbb{R}_{+}^{d}\right)}^{2} \leqslant C\|u\|_{L^{2}\left(\mathbb{R}_{+}^{d}\right)}\left(\|u\|_{L^{2}\left(\mathbb{R}_{+}^{d}\right)}+\|\operatorname{div} u\|_{L^{2}\left(\mathbb{R}_{+}^{d}\right)}\right),
$$

for $u \in C^{1}\left(\mathbb{R}^{d}\right)$ with support in the unit ball, where $\mathbb{R}_{+}^{d}:=\mathbb{R}^{d-1} \times(0,+\infty)$. Define, for $\left(\xi^{\prime}, x_{d}\right) \in \mathbb{R}^{d-1} \times \mathbb{R}$

$$
\mathcal{F} v\left(\xi^{\prime}, x_{d}\right):=\int_{\mathbb{R}^{d-1}} v\left(x^{\prime}, x_{d}\right) e^{-i x^{\prime} \cdot \xi^{\prime}} d x^{\prime} \text { for } v \in C_{c}^{1}\left(\mathbb{R}^{d}\right)
$$

We have

$$
\begin{aligned}
& \|u \cdot \nu\|_{H^{-1 / 2}\left(\partial \mathbb{R}_{+}^{d}\right)}^{2}=\int_{\mathbb{R}^{d-1}}\left|\mathcal{F} u_{d}\left(\xi^{\prime}, 0\right)\right|^{2}\left(1+\left|\xi^{\prime}\right|^{2}\right)^{-1 / 2} d \xi^{\prime} \\
& \quad \leqslant \frac{1}{2} \int_{0}^{\infty} \int_{\mathbb{R}^{d-1}}\left|\partial_{x_{d}} \mathcal{F} u_{d}\left(\xi^{\prime}, x_{d}\right)\right|\left|\mathcal{F} u_{d}\left(\xi^{\prime}, x_{d}\right)\right|\left(1+\left|\xi^{\prime}\right|^{2}\right)^{-1 / 2} d \xi^{\prime} d x_{d} .
\end{aligned}
$$

Using Hölder's inequality, we obtain

$\|u \cdot \nu\|_{H^{-1 / 2}\left(\partial \mathbb{R}_{+}^{d}\right)}^{2} \leqslant \frac{1}{2}\left(\int_{\mathbb{R}_{+}^{d}}\left|\partial_{x_{d}} \mathcal{F} u_{d}\left(\xi^{\prime}, 0\right)\right|^{2}\left(1+\left|\xi^{\prime}\right|^{2}\right)^{-1} d \xi^{\prime} d x_{d}\right)^{1 / 2}\left(\int_{\mathbb{R}_{+}^{d}}\left|\mathcal{F} u_{d}\left(\xi^{\prime}, 0\right)\right|^{2} d \xi^{\prime} d x_{d}\right)^{1 / 2}$.

Since

$\left|\partial_{x_{d}} \mathcal{F} u_{d}\left(\xi^{\prime}, x_{d}\right)\right|=\left|\mathcal{F}(\operatorname{div} u)\left(\xi^{\prime}, x_{d}\right)-\sum_{j=1}^{d-1} i \xi_{j}^{\prime} \mathcal{F} u_{j}\left(\xi^{\prime}, x_{d}\right)\right| \leqslant\left|\mathcal{F}(\operatorname{div} u)\left(\xi^{\prime}, x_{d}\right)\right|+\sum_{j=1}^{d-1}\left|\xi_{j}^{\prime} \mathcal{F} u_{j}\left(\xi^{\prime}, x_{d}\right)\right|$,

it follows from Parseval's theorem that

$$
\int_{\mathbb{R}_{+}^{d}}\left|\partial_{x_{d}} \mathcal{F} u_{d}\left(\xi^{\prime}, x_{d}\right)\right|^{2}\left(1+\left|\xi^{\prime}\right|^{2}\right)^{-1} d \xi^{\prime} d x_{d} \leqslant C \int_{\mathbb{R}_{+}^{d}}\left(|\operatorname{div} u(x)|^{2}+|u(x)|^{2}\right) d x .
$$

By Parseval's theorem, we also have

$$
\int_{\mathbb{R}_{+}^{d}}\left|\mathcal{F} u_{d}\left(\xi^{\prime}, x_{d}\right)\right|^{2} d \xi^{\prime} d x_{d} \leqslant C \int_{\mathbb{R}_{+}^{d}}|u(x)|^{2} d x
$$

Combining (3.13), (3.14), and (3.15) yields (3.12).

As a consequence of Lemma 3.2, one can derive the following compactness result for Maxwell's equations, which is the key ingredient in the proof of Theorem 2.1.

Lemma 3.5. Let $\Omega \subset \mathbb{R}^{3}$ be open, bounded, and of class $C^{1}$ and let $\varepsilon, \hat{\varepsilon}, \mu, \hat{\mu}$ be real, symmetric, uniformly elliptic matrix-valued functions defined in $\Omega$ and of class $C^{1}$. Let $\left(J_{e, n}\right),\left(J_{m, n}\right),\left(\hat{J}_{e, n}\right)$, and $\left(\hat{J}_{m, n}\right) \subset\left[L^{2}(\Omega)\right]^{3}$, and let $\left(E_{n}, H_{n}\right),\left(\hat{E}_{n}, \hat{H}_{n}\right) \in[H(\operatorname{curl}, \Omega)]^{2}$ be such that

$$
\left\{\begin{array} { c l } 
{ \nabla \times E _ { n } = i \omega \mu H _ { n } + J _ { e , n } } & { \text { in } \Omega , } \\
{ \nabla \times H _ { n } = - i \omega \varepsilon E _ { n } + J _ { m , n } } & { \text { in } \Omega , }
\end{array} \quad \left\{\begin{array}{cl}
\nabla \times \hat{E}_{n}=i \omega \hat{\mu} \hat{H}_{n}+\hat{J}_{e, n} & \text { in } \Omega \\
\nabla \times \hat{H}_{n}=-i \omega \hat{\varepsilon} \hat{E}_{n}+\hat{J}_{m, n} & \text { in } \Omega
\end{array}\right.\right.
$$

and

$$
E_{n} \times \nu=\hat{E}_{n} \times \nu \quad \text { and } \quad H_{n} \times \nu=\hat{H}_{n} \times \nu \text { on } \partial \Omega .
$$


Let $S$ be a connected component of $\partial \Omega$ and assume that

$(\varepsilon, \hat{\varepsilon})(x),(\mu, \hat{\mu})(x)$ satisfy the complementing condition with respect to $\nu(x)$ for all $x \in S$,

$$
\left(E_{n}, H_{n}, \hat{E}_{n}, \hat{H}_{n}\right) \text { are bounded in }\left[L^{2}(\Omega)\right]^{12} \text {, }
$$

and

$$
\left(J_{e, n}, J_{m, n}, \hat{J}_{e, n}, \hat{J}_{m, n}\right) \text { converges in }\left[L^{2}(\Omega)\right]^{12} \text { as } n \rightarrow+\infty .
$$

There exists a neighborhood $U$ of $S$ such that, up to a subsequence, $\left(E_{n}, H_{n}, \hat{E}_{n}, \hat{H}_{n}\right)$ converges in $\left[L^{2}(U \cap \Omega)\right]^{12}$.

Proof. Let $\ell \geqslant 1, x_{k} \in S, r_{k}>0$, and $\varphi_{k} \in C^{1}\left(\mathbb{R}^{3}\right)$ for $1 \leqslant k \leqslant \ell$ be such that $B_{r_{k}}\left(x_{k}\right) \cap \Omega$ is simply connected with connected boundary, supp $\varphi_{k} \Subset B_{r_{k} / 2}\left(x_{k}\right)$, and $\sum_{k=1}^{\ell} \varphi=1$ in a neighborhood $U$ of $S$. For $1 \leqslant k \leqslant \ell$, set, $O_{k}=B_{r_{k}}\left(x_{k}\right) \cap \Omega$, and, in $O_{k}$, define

$$
\mathbf{E}_{k, n}=T\left(\varphi_{k} E_{n}\right), \quad \hat{\mathbf{E}}_{k, n}=T\left(\varphi_{k} \hat{E}_{n}\right), \quad \mathbf{H}_{k, n}=T\left(\varphi_{k} H_{n}\right), \quad \hat{\mathbf{H}}_{k, n}=T\left(\varphi_{k} \hat{H}_{n}\right),
$$

where $T$ is the operator given in Lemma 3.1 for the set $O_{k}$. By Lemma 3.1, we have

$$
\left\|\left(\mathbf{E}_{k, n}, \hat{\mathbf{E}}_{k, n}, \mathbf{H}_{k, n}, \hat{\mathbf{H}}_{k, n}\right)\right\|_{H^{1}\left(O_{k}\right)} \leqslant C\left\|\left(E_{n}, \hat{E}_{n}, H_{n}, \hat{H}_{n}, J_{e, n}, \hat{J}_{e, n}, J_{m, n}, \hat{J}_{m, n}\right)\right\|_{L^{2}\left(O_{k}\right)} .
$$

Without loss of generality, one may assume that, as $n \rightarrow+\infty$,

$$
\left(\mathbf{E}_{k, n}, \hat{\mathbf{E}}_{k, n}, \mathbf{H}_{k, n}, \hat{\mathbf{H}}_{k, n}\right) \text { converges in }\left[L^{2}\left(O_{k}\right)\right]^{12},
$$$$
\left(\mathbf{E}_{k, n}-\hat{\mathbf{E}}_{k, n}, \mathbf{H}_{k, n}-\hat{\mathbf{H}}_{k, n}\right) \text { converges in }\left[H^{-1 / 2}\left(\partial O_{k}\right)\right]^{6},
$$

and, by Lemma 3.3 ,

$$
\left(\varphi_{k} E_{n}-\mathbf{E}_{k, n}, \varphi_{k} \hat{E}_{k, n}-\hat{\mathbf{E}}_{k, n}, \varphi_{k} H_{n}-\mathbf{H}_{k, n}, \varphi_{k} \hat{H}_{n}-\hat{\mathbf{H}}_{k, n}\right) \text { converges in }\left[\left[H^{1}\left(O_{k}\right)\right]^{*}\right]^{12} \text {. }
$$

Applying Lemma 3.2 , we have

$$
\begin{aligned}
\left\|\left(\left(\varphi_{k} E_{n}-\mathbf{E}_{k, n}\right)-\left(\varphi_{k} E_{l}-\mathbf{E}_{k, l}\right),\left(\varphi_{k} \hat{E}_{n}-\hat{\mathbf{E}}_{k, n}\right)-\left(\varphi_{k} \hat{E}_{l}-\hat{\mathbf{E}}_{l}\right)\right)\right\|_{L^{2}\left(O_{k}\right)} \\
\leqslant C\left\|\left(\left(\varphi_{k} E_{n}-\mathbf{E}_{k, n}\right)-\left(\varphi_{k} E_{l}-\mathbf{E}_{k, l}\right),\left(\varphi_{k} \hat{E}_{n}-\hat{\mathbf{E}}_{k, n}\right)-\left(\varphi_{k} \hat{E}_{l}-\hat{\mathbf{E}}_{k, l}\right)\right)\right\|_{\left[H^{1}\left(O_{k}\right)\right]^{*}} \\
+C\left\|\left(\mathbf{E}_{k, n}-\mathbf{E}_{k, l}, \hat{\mathbf{E}}_{k, n}-\hat{\mathbf{E}}_{k, l}, J_{m, n}-J_{m, l}, \hat{J}_{m, n}-\hat{J}_{m, l}\right)\right\|_{L^{2}\left(O_{k}\right)} \\
\quad+C\left\|\left(\mathbf{E}_{k, n}-\hat{\mathbf{E}}_{k, n}\right)-\left(\mathbf{E}_{l, n}-\hat{\mathbf{E}}_{l, n}\right)\right\|_{H^{-1 / 2}\left(\partial O_{k}\right)} \rightarrow 0 \text { as } l, n \rightarrow+\infty
\end{aligned}
$$

and

$$
\begin{gathered}
\left\|\left(\left(\varphi_{k} H_{n}-\mathbf{H}_{k, n}\right)-\left(\varphi_{k} H_{l}-\mathbf{H}_{k, l}\right),\left(\varphi_{k} \hat{H}_{n}-\hat{\mathbf{H}}_{k, n}\right)-\left(\varphi_{k} \hat{H}_{l}-\hat{\mathbf{H}}_{l}\right)\right)\right\|_{L^{2}\left(O_{k}\right)} \\
\leqslant C\left\|\left(\left(\varphi_{k} H_{n}-\mathbf{H}_{k, n}\right)-\left(\varphi_{k} H_{l}-\mathbf{H}_{k, l}\right),\left(\varphi_{k} \hat{H}_{n}-\hat{\mathbf{H}}_{k, n}\right)-\left(\varphi_{k} \hat{H}_{l}-\hat{\mathbf{H}}_{k, l}\right)\right)\right\|_{\left[H^{1}\left(O_{k}\right)\right]^{*}} \\
+C\left\|\left(\mathbf{H}_{k, n}-\mathbf{H}_{k, l}, \hat{\mathbf{H}}_{k, n}-\hat{\mathbf{H}}_{k, l}, J_{e, n}-J_{e, l}, \hat{J}_{e, n}-\hat{J}_{e, l}\right)\right\|_{L^{2}\left(O_{k}\right)} \\
\quad+C\left\|\left(\mathbf{H}_{k, n}-\hat{\mathbf{H}}_{k, n}\right)-\left(\mathbf{H}_{l, n}-\hat{\mathbf{H}}_{l, n}\right)\right\|_{H^{-1 / 2}\left(\partial O_{k}\right)} \rightarrow 0 \text { as } l, n \rightarrow+\infty .
\end{gathered}
$$

Hence $\left(\varphi_{k} E_{k, n}, \varphi_{k} \hat{E}_{k, n}, \varphi_{k} H_{k, n}, \varphi_{k} \hat{H}_{k, n}\right)$ is a Cauchy sequence in $\left[L^{2}\left(O_{k}\right)\right]^{12}$ and therefore convergence in $\left[L^{2}\left(O_{k}\right)\right]^{12}$. The conclusion then follows since $\sum_{k=1}^{\ell} \varphi_{k}=1$ in $U$.

We next recall the following well-posedness result on (1.3) (see [25, Lemma 6]).

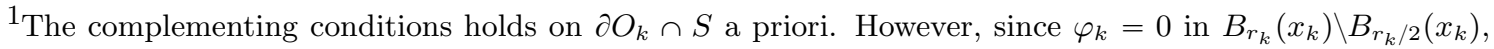
one can modify $\varepsilon, \mu, \hat{\varepsilon}, \hat{\mu}$ in $B_{r_{k}}\left(x_{k}\right) \backslash B_{2 r_{k} / 3}\left(x_{k}\right)$ so that the complementing conditions hold on the whole boundary and the systems are unchanged, e.g. $\varepsilon>\hat{\varepsilon}$ and $\mu>\hat{\mu}$ in $B_{r_{k}}\left(x_{k}\right) \backslash B_{r_{k} / 2}\left(x_{k}\right)$ (see Proposition 2.1).
} 
Lemma 3.6. Let $0<\delta<1, f, g \in\left[L^{2}\left(\mathbb{R}^{3}\right)\right]^{3}$, and $\left(\varepsilon_{\delta}, \mu_{\delta}\right)$ be defined in (1.1). Assume that supp $f$, supp $g \subset B_{R_{0}}$. There exists a unique radiating solution $\left(\mathcal{E}_{\delta}, \mathcal{H}_{\delta}\right) \in\left[H_{\text {loc }}\left(\text { curl, } \mathbb{R}^{3}\right)\right]^{2}$ to

Moreover, we have

$$
\left\{\begin{array}{lll}
\nabla \times \mathcal{E}_{\delta} & =i \omega \mu_{\delta} \mathcal{H}_{\delta}+f & \text { in } \mathbb{R}^{3}, \\
\nabla \times \mathcal{H}_{\delta} & =-i \omega \varepsilon_{\delta} \mathcal{E}_{\delta}+g & \text { in } \mathbb{R}^{3}
\end{array}\right.
$$

$$
\left\|\left(\mathcal{E}_{\delta}, \mathcal{H}_{\delta}\right)\right\|_{H\left(\operatorname{curl}, B_{R}\right)} \leqslant \frac{C_{R}}{\delta}\|(f, g)\|_{L^{2}}
$$

where $C_{R}$ denotes a positive constant depending on $R, R_{0}, \varepsilon$, and $\mu$, but independent of $f$, $g$, and $\delta$.

We now deal with the uniqueness of (1.3) for $\delta=0$.

Lemma 3.7. Let $J=0$ and $\left(E_{0}, H_{0}\right) \in\left[H_{\text {loc }}\left(\text { curl, } \mathbb{R}^{3}\right)\right]^{2}$ is a radiating solution of (1.3) with $\delta=0$. Then

$$
E_{0}=H_{0}=0 \text { in } \mathbb{R}^{3} .
$$

Proof. The proof is quite standard as in the usual case. We present it here for the completeness. Multiplying the equation of $E_{0}$ by $\nabla \times \bar{E}_{0}\left(\bar{E}_{0}\right.$ denotes the conjugate of $\left.E_{0}\right)$, we have

$$
\int_{B_{R_{0}}}\left\langle\mu^{-1} \nabla \times E_{0}, \nabla \times E_{0}\right\rangle=\int_{B_{R_{0}}}\left\langle i \omega H_{0}, \nabla \times E_{0}\right\rangle .
$$

Integrating by parts the RHS and using the equation of $H_{0}$, we obtain

$$
\int_{B_{R_{0}}}\left\langle\mu^{-1} \nabla \times E_{0}, \nabla \times E_{0}\right\rangle=\int_{B_{R_{0}}} \omega^{2}\left|E_{0}\right|^{2}+\int_{\partial B_{R_{0}}} i \omega\left(H_{0} \times \nu\right) \cdot \bar{E}_{0} .
$$

This implies

$$
\Re \int_{\partial B_{R_{0}}}\left(H_{0} \times \nu\right) \cdot \bar{E}_{0}=0 .
$$

It follows, see, e.g., [8, Theorem 6.10], that $E_{0}=H_{0}=0$ in $\mathbb{R}^{3} \backslash B_{R_{0}}$. Therefore, $E_{0}=H_{0}=0$ in $\mathbb{R}^{3}$ by the unique continuation principle, see [4, 31] (see also [16, 35]).

3.2. Proof of Theorem 2.1. We first prove (2.1) with $r_{0}:=R_{0}+1$ by contradiction. Assume that there exist a sequence $\left(\delta_{n}\right) \subset(0,1)$ and a sequence $\left(J_{n}\right) \subset\left[L^{2}\left(\mathbb{R}^{3}\right)\right]^{3}$ with $\operatorname{supp} J_{n} \subset B_{R_{0}}$ such that

$$
\left\|\left(E_{n}, H_{n}\right)\right\|_{L^{2}\left(B_{r_{0}}\right)}=1 \quad \text { and } \quad \lim _{n \rightarrow+\infty}\left\|J_{n}\right\|_{L^{2}\left(\mathbb{R}^{3}\right)}=0 .
$$

Here $\left(E_{n}, H_{n}\right)$ is the corresponding solution of (1.3) with $\delta=\delta_{n}$ and $J=J_{n}$. By Lemma 3.6. without loss of generality, one may assume that $\delta_{n} \rightarrow 0$. Since

$$
\left\{\begin{array}{cl}
\nabla \times E_{n}=i \omega H_{n} & \text { in } \mathbb{R}^{3} \backslash B_{R_{0}}, \\
\nabla \times H_{n}=-i \omega E_{n} & \text { in } \mathbb{R}^{3} \backslash B_{R_{0}},
\end{array}\right.
$$

we have, for $R>R_{0}+1 / 2$, by [20, Lemma 5],

$\left\|\left(E_{n}, H_{n}\right)\right\|_{L^{2}\left(B_{R} \backslash B_{R_{0}+1 / 2}\right)} \leqslant C_{R}\left\|\left(\nu \times E_{n}, \nu \times H_{n}\right)\right\|_{H^{-1 / 2}\left(\operatorname{div}_{\Gamma}, \partial B_{R_{0}+1 / 2}\right)}$

$$
\leqslant C_{R}\left\|\left(E_{n}, H_{n}\right)\right\|_{H\left(\operatorname{curl}, B_{R_{0}+1 / 2} \backslash B_{R_{0}}\right)} \text { by the trace theory (see e.g. [12]). }
$$

Combining (3.16) and (3.17) yields

$$
\left\|\left(E_{n}, H_{n}\right)\right\|_{L^{2}\left(B_{R}\right)} \leqslant C_{R} \quad \text { for all } R>0 .
$$


Fix $\psi \in C_{c}^{1}\left(\mathbb{R}^{3}\right)$ (arbitrary) be such that $\psi=0$ in a neighborhood of $\Gamma$. We have, in $\mathbb{R}^{3}$,

$\nabla \times\left(\psi E_{n}\right)=\psi \nabla \times E_{n}+\nabla \psi \times E_{n} \quad$ and $\quad \operatorname{div}\left(\varepsilon_{0} \psi E_{n}\right)=\psi \operatorname{div}\left(\varepsilon_{0} E_{n}\right)+\nabla \psi \cdot \varepsilon_{0} E_{n}$.

Using (3.18) and the system of equations of $\left(E_{n}, H_{n}\right)$ and applying e.g. [25, Lemma 1], one may assume that

$$
\left(\psi E_{n}\right) \text { converges in } L^{2}\left(\mathbb{R}^{3}\right),
$$

which yields, since $\psi$ is arbitrary,

$$
\left(E_{n}\right) \text { converges in } L_{\mathrm{loc}}^{2}\left(\mathbb{R}^{3} \backslash \Gamma\right) \text {. }
$$

Similarly, one may also assume that

$$
\left(H_{n}\right) \text { converges in } L_{\text {loc }}^{2}\left(\mathbb{R}^{3} \backslash \Gamma\right) \text {. }
$$

For $\tau>0$ sufficiently small (the smallness depends only on $D$ ), define $\mathcal{F}: D_{\tau} \rightarrow D_{-\tau}$ by

$$
\mathcal{F}\left(x_{\Gamma}+t \nu\left(x_{\Gamma}\right)\right)=x_{\Gamma}-t \nu\left(x_{\Gamma}\right) \quad \forall x_{\Gamma} \in \Gamma, t \in(-\tau, 0)
$$

and set, in $D_{-\tau}$,

$$
(\hat{\varepsilon}, \hat{\mu})=\left(\mathcal{F}_{*} \varepsilon^{-}, \mathcal{F}_{*} \mu^{-}\right) \quad \text { and } \quad\left(\hat{E}_{n}, \hat{H}_{n}\right)=\left(\mathcal{F} * E_{n}, \mathcal{F} * H_{n}\right) .
$$

Note that the pairs $(\varepsilon, \hat{\varepsilon})(x)$ and $(\mu, \hat{\mu})(x)$ satisfy the complementing conditions with respect to $\nu(x)$ for all $x \in \Gamma$ if and only if the pairs $\left(\varepsilon^{+},-\varepsilon^{-}\right)(x)$ and $\left(\mu^{+},-\mu^{-}\right)(x)$ do, by Proposition 2.1. By a change of variables for the Maxwell equations, see e.g. [25, Lemma 7], we have

$$
\left\{\begin{array} { c l } 
{ \nabla \times E _ { n } = i \omega \mu H _ { n } } & { \text { in } D _ { - \tau } , } \\
{ \nabla \times H _ { n } = - i \omega \varepsilon E _ { n } + J _ { n } } & { \text { in } D _ { - \tau } , }
\end{array} \quad \left\{\begin{array}{cl}
\nabla \times \hat{E}_{n}=i \omega \hat{\mu} \hat{H}_{n}+\hat{J}_{e, n} & \text { in } D_{-\tau}, \\
\nabla \times \hat{H}_{n}=-i \omega \hat{\varepsilon} \hat{E}_{n}+\hat{J}_{m, n} & \text { in } D_{-\tau},
\end{array}\right.\right.
$$

and

$$
E_{n} \times \nu=\hat{E}_{n} \times \nu \quad \text { and } \quad H_{n} \times \nu=\hat{H}_{n} \times \nu \quad \text { on } \Gamma \text {. }
$$

Here

$$
\hat{J}_{e, n}=-\delta \omega \mathcal{F}_{*} I \hat{H}_{n} \quad \text { and } \quad \hat{J}_{m, n}=\delta \omega \mathcal{F}_{*} I \hat{E}_{n}+\mathcal{F}_{*} J_{n} \quad \text { in } D_{-\tau} .
$$

By Lemma 3.5, there exists a neighborhood $U$ of $\Gamma$ such that, up to a subsequence,

$$
\left(E_{n}, H_{n}, \hat{E}_{n}, \hat{H}_{n}\right) \text { converges in }\left[L^{2}\left(U \cap D_{-\tau / 2}\right)\right]^{12} \text {. }
$$

It follows from (3.19), (3.20), and (3.21) that, up to a subsequence,

$$
\left(E_{n}, H_{n}\right) \text { converges in }\left[L_{\text {loc }}^{2}\left(\mathbb{R}^{3}\right)\right]^{12} \text {. }
$$

Moreover, the limit $\left(E_{0}, H_{0}\right)$ is in $\left[H_{\text {loc }}\left(\operatorname{curl}, \mathbb{R}^{3}\right)\right]^{2}$ and is a radiating solution of the equations

$$
\left\{\begin{array}{cl}
\nabla \times E_{0}=i \omega \mu_{0} H & \text { in } \mathbb{R}^{3} \\
\nabla \times H_{0}=-i \omega \varepsilon_{0} E & \text { in } \mathbb{R}^{3} .
\end{array}\right.
$$

By Lemma 3.7, we have

$$
E_{0}=H_{0}=0 \text { in } \mathbb{R}^{3} \text {. }
$$

This contradicts the fact that $\left\|\left(E_{n}, H_{n}\right)\right\|_{L^{2}\left(B_{r_{0}}\right)}=1$ and, up to a subsequence, $\left(E_{n}, H_{n}\right)$ converges to $\left(E_{0}, H_{0}\right)$ in $\left[L_{\text {loc }}^{2}\left(\mathbb{R}^{3}\right)\right]^{6}$.

Hence (2.1) holds for $R=R_{0}+1$. This implies, exactly as in the proof of (3.18),

$$
\left\|\left(E_{\delta}, H_{\delta}\right)\right\|_{L^{2}\left(B_{R}\right)} \leqslant C_{R}\|J\|_{L^{2}\left(\mathbb{R}^{3}\right)} \quad \text { for all } R>R_{0}+1,
$$

which is (2.1).

We next establish the remaining part of Theorem 2.1. By (2.1), for every sequence $\left(\delta_{n}\right) \rightarrow$ 0 , there exists a subsequence $\left(\delta_{n_{k}}\right)$ such that $\left(E_{\delta_{n_{k}}}, H_{\delta_{n_{k}}}\right)$ converges weakly in $\left[L_{\text {loc }}^{2}\left(\mathbb{R}^{3}\right)\right]^{6}$. Moreover, the limit $\left(E_{0}, H_{0}\right) \in\left[H_{\text {loc }}\left(\operatorname{curl}, \mathbb{R}^{3}\right)\right]^{2}$ is a radiating solution of (1.3) with $\delta=0$. 
By Lemma 3.7, the limit is unique. Therefore, $\left(E_{\delta}, H_{\delta}\right)$ converges to $\left(E_{0}, H_{0}\right)$ weakly in $\left[L_{\text {loc }}^{2}\left(\mathbb{R}^{3}\right)\right]^{6}$ as $\delta \rightarrow 0$. It is then clear that (2.2) follows from (2.1). The strong convergence follows from (2.1) by the same way (3.22) followed from (3.18) and once again using the uniqueness of the limit. The proof is complete.

A key part in the proof of Theorem 2.1] is the compactness result in Lemma 3.5 which is derived from Lemma 3.2 where the complementing conditions plays a crucial role. Assume that $(\varepsilon, \hat{\varepsilon})$ and $(\mu, \hat{\mu})$ both satisfy the complementing conditions and of class $C^{2}$. Then, instead of (3.1) and (3.2), one has (see [2])

$$
\|(E, \hat{E}, H, \hat{H})\|_{H^{1}(\Omega)} \leqslant C\left(\|(E, \hat{E}, H, \hat{H})\|_{L^{2}(\Omega)}+\left\|\left(J_{e}, \hat{J}_{e}, J_{m}, \hat{J}_{m}\right)\right\|_{H(\operatorname{div}, \Omega)}\right)
$$

for some positive constant $C$ independent of $(E, \hat{E}, H, \hat{H}),\left(J_{e}, \hat{J}_{e}, J_{m}, \hat{J}_{m}\right)$. The following proposition shows that the complementing conditions on each pair $(\varepsilon, \hat{\varepsilon})$ and $(\mu, \hat{\mu})$ are necessary to have (3.23).

Proposition 3.1. Let $\Omega$ be a bounded, connected open subset of $\mathbb{R}^{3}$ and of class $C^{2}$ and let $\varepsilon, \hat{\varepsilon}, \mu, \hat{\mu}$ be real, symmetric, uniformly elliptic matrix-valued functions defined in $\Omega$ and of class $C^{1}$. Assume that either $(\varepsilon, \hat{\varepsilon})$ or $(\mu, \hat{\mu})$ does not satisfy the complementing condition at some point $x_{0} \in \partial \Omega$. Then there exist sequences $\left(J_{e, n}\right),\left(J_{m, n}\right),\left(\hat{J}_{e, n}\right),\left(\hat{J}_{m, n}\right) \subset H(\operatorname{div}, \Omega)$ and $\left(\left(E_{n}, H_{n}\right)\right),\left(\left(\hat{E}_{n}, \hat{H}_{n}\right)\right) \subset\left[H^{1}(\Omega)\right]^{6}$ such that

$$
\begin{gathered}
\left\{\begin{array} { c c } 
{ \nabla \times E _ { n } = i \omega \mu H _ { n } + J _ { e , n } \quad \text { in } \Omega , } \\
{ \nabla \times H _ { n } = - i \omega \varepsilon E _ { n } + J _ { m , n } \quad \text { in } \Omega , }
\end{array} \quad \left\{\begin{array}{cc}
\nabla \times \hat{E}_{n}=i \omega \hat{\mu} \hat{H}_{n}+\hat{J}_{e, n} & \text { in } \Omega, \\
\nabla \times \hat{H}_{n}=-i \omega \hat{\varepsilon} \hat{E}_{n}+\hat{J}_{m, n} & i n \Omega,
\end{array}\right.\right. \\
E_{n} \times \nu=\hat{E}_{n} \times \nu, \quad H_{n} \times \nu=\hat{H}_{n} \times \nu \text { on } \partial \Omega
\end{gathered}
$$

and

$$
\sup _{n}\left\|\left(E_{n}, \hat{E}_{n}, H_{n}, \hat{H}_{n}\right)\right\|_{H^{1}(\Omega)}=+\infty .
$$

The proof of Proposition 3.1 is given in the appendix.

\section{VARiational approach For the CAUChy PROBlems}

This section containing three subsections is devoted to Theorem 2.2. In the first section, we present lemmas used in the proof of Theorem 2.2. The proof of Theorem 2.2 is given in the second subsection. In the last section, we give some applications of Theorem 2.2 ,

4.1. Some useful lemmas. We begin with the following version of Poincaré's lemma which plays an important role in the proof of Theorem 2.2 .

Lemma 4.1. Let $0 \leqslant \alpha<2$ and $\Omega$ be a domain diffeomorphic to $B_{1}$. There exists a linear operator

$$
T_{1}:\{f \in H(\operatorname{div}, \Omega) ; \operatorname{div} f=0 \text { in } \Omega\} \rightarrow H(\operatorname{curl}, \Omega)
$$

such that

$$
\nabla \times T_{1}(f)=f \text { in } \Omega
$$

and

$$
\int_{\Omega}\left|T_{1}(f)\right|^{2} d x \leqslant C \int_{\Omega} d_{\partial \Omega}^{\alpha}|f|^{2} d x
$$

for some positive constant $C=C(\alpha, \Omega)$ independent of $f$. 
Proof. We first consider the case $\Omega=B_{1}$. Without loss of generality, one can assume that $1<\alpha<2$. We first assume that $f$ is smooth (and $\operatorname{div} f=0$ ). Set

$$
F(x)=-\int_{0}^{1} t x \times f(t x) d t \text { in } B_{1} .
$$

Using the fact that, for two vector fields $A$ and $B$ defined in $B_{1}$,

$$
\nabla \times(A \times B)=A \operatorname{div} B-B \operatorname{div} A+(B \cdot \nabla) A-(A \cdot \nabla) B,
$$

we have, in $B_{1}$,

$$
\nabla \times F(x)=2 \int_{0}^{1} t f(t x) d t+\int_{0}^{1} t(x \cdot \nabla) f(t x) d t=2 \int_{0}^{1} t f(t x) d t+\int_{0}^{1} t^{2} \frac{d}{d t}[f(t x)] d t=f(x) .
$$

We next prove (4.1). We have

$$
\begin{aligned}
& \int_{B_{1}}|F|^{2} d x \stackrel{(4.2)}{\leqslant} \int_{B_{1}}\left(\int_{0}^{1}|t x||f(t x)| d t\right)^{2} d x \\
& \stackrel{\text { Hölder }}{\leqslant} \int_{B_{1}}\left(\int_{0}^{1}|t x|^{2}|f(t x)|^{2}(1-|t x|)^{\alpha} d t \int_{0}^{1}(1-t|x|)^{-\alpha} d t\right) d x .
\end{aligned}
$$

In what follows in this proof, $C$ denotes a positive constant depending only on $\alpha$ and $\Omega$. Since

it follows that

$$
\int_{0}^{1}(1-t|x|)^{-\alpha} d t=(\alpha-1)|x|^{-1}\left[(1-|x|)^{-\alpha+1}-1\right] \leqslant C(1-|x|)^{-\alpha+1},
$$

$$
\begin{aligned}
& \int_{B_{1}}|F|^{2} d x \leqslant C \int_{B_{1}} \int_{0}^{1}|t x|^{2}|f(t x)|^{2}(1-|t x|)^{\alpha} d t(1-|x|)^{-\alpha+1} d x \\
& \stackrel{y=t x}{\leqslant} C \int_{0}^{1} \int_{B_{t}}|y|^{2}|f(y)|^{2}(1-|y|)^{\alpha}(t-|y|)^{-\alpha+1} t^{\alpha-4} d y d t \\
& \stackrel{\text { Fubini }}{\leqslant} C \int_{B_{1}}|y|^{2}|f(y)|^{2}(1-|y|)^{\alpha} \int_{y}^{1}(t-|y|)^{-\alpha+1} t^{\alpha-4} d t d y .
\end{aligned}
$$

Since $\alpha<2$, we have

$$
\begin{aligned}
\int_{y}^{1}(t-|y|)^{-\alpha+1} t^{\alpha-4} d t & =\left.\frac{1}{2-\alpha}(t-|y|)^{-\alpha+2} t^{\alpha-4}\right|_{y} ^{1}+\frac{1}{(2-\alpha)(4-\alpha)} \int_{y}^{1}(t-|y|)^{-\alpha+2} t^{\alpha-5} d t \\
& \leqslant C+C \int_{y}^{1} t^{-3} \leqslant C y^{-2}
\end{aligned}
$$

Combining (4.3) and (4.4) yields

$$
\int_{B_{1}}|F|^{2} d x \leqslant C \int_{B_{1}}|f(y)|^{2}(1-|y|)^{\alpha} d y
$$

which is (4.6). The conclusion for a general $f$ now follows by a standard approximation process.

We now consider a general $\Omega$. Let $\mathcal{T}: \Omega \rightarrow B_{1}$ be a diffeomorphism. Set, for $x^{\prime} \in B_{1}$,

$$
f^{\prime}\left(x^{\prime}\right)=\frac{\nabla \mathcal{T}(x)}{J(x)} f(x) \text { with } x^{\prime}=\mathcal{T}(x) \text { and } J(x)=\operatorname{det} \mathcal{T}(x)
$$


and

Since (see, e.g., [18, Lemma 3.59]),

$$
F^{\prime}\left(x^{\prime}\right)=-\int_{0}^{1} t x^{\prime} \times f^{\prime}\left(t x^{\prime}\right) d t .
$$

$$
\nabla^{\prime} \cdot f^{\prime}\left(x^{\prime}\right)=\frac{\nabla \cdot f(x)}{J(x)}=0 \text { in } B_{1},
$$

it follows from the case $\Omega=B_{1}$ that

$$
\nabla^{\prime} \times F^{\prime}\left(x^{\prime}\right)=f^{\prime}\left(x^{\prime}\right) \text { in } B_{1} .
$$

Set, for $x \in \Omega$,

$$
T_{1}(f)(x)=\nabla \mathcal{T}^{T}(x) F^{\prime}\left(x^{\prime}\right) \text { with } x^{\prime}=\mathcal{T}(x) .
$$

Then (see, e.g., [18, Corollary 3.58])

$$
\nabla \times T_{1}(f)(x)=f(x) \text { for } x \in \Omega .
$$

Assertion (4.6) now follows from (4.5).

Remark 4.1. The definition of $F$ in (4.2) is an explicit formula for solving the Poincaré lemma in a star-shaped domain taken from a note of Dacorogna [1].

In what follows, we denote $\mathbb{R}_{+}^{3}=\left\{\left(x_{1}, x_{2}, x_{3}\right) \in \mathbb{R}^{3} ; x_{3}>0\right\}, \mathbb{R}_{0}^{3}=\left\{\left(x_{1}, x_{2}, x_{3}\right) \in \mathbb{R}^{3} ; x_{3}=\right.$ $0\}, Q=(-1,1)^{3}, Q_{+}=Q \cap \mathbb{R}_{+}^{3}$, and $Q_{0}=Q \cap \mathbb{R}_{0}^{3}$. The proof of Lemma 4.1 can be used to obtain

Lemma 4.2. Let $0 \leqslant \alpha<2$ and let $\Omega$ be an open bounded subset of $\mathbb{R}^{3}$ and let $\mathcal{H}: \Omega \rightarrow Q_{+}$ be a diffeomorphism. Denote $S=\partial Q_{+} \backslash Q_{0}$ and let $U \subset \mathbb{R}^{3}$ be a neighborhood of $\bar{S}$. There exist an open set $V \subset \mathbb{R}^{3}$ containing $\mathcal{H}^{-1}(\bar{S})$ and a linear operator

$$
T_{1}:\{f \in H(\operatorname{div}, \Omega) ; \operatorname{div} f=0 \text { in } \Omega\} \rightarrow H(\operatorname{curl}, \Omega)
$$

such that

$$
\begin{gathered}
\nabla \times T_{1}(f)=f \text { in } \Omega, \\
\int_{\Omega}\left|T_{1}(f)\right|^{2} d x \leqslant C \int_{\Omega} d_{\partial \Omega}^{\alpha}|f|^{2} d x,
\end{gathered}
$$

for some positive constant $C=C(\alpha, \Omega)$ independent of $f$, and

$$
T_{1}(f)=0 \text { in } V \cap \Omega \text { if } f=0 \text { in } \mathcal{H}^{-1}\left(U \cap Q_{+}\right) \text {. }
$$

Proof. Without loss of generality, one can assume that $1<\alpha<2$. Let $\mathcal{T}_{1}: Q_{+} \rightarrow B_{1}$ be a bi-Lipschitz homeomorphism such that $\mathcal{T}_{1}(S)=\Sigma$ and $\overline{\mathcal{C}} \subset \mathcal{T}_{1}\left(U \cap Q_{+}\right)$, where $\Sigma:=\partial B_{1} \cap \overline{\mathbb{R}}_{+}^{3}$ and $\mathcal{C}:=\{r \sigma ; r \in(0,1)$ and $\sigma \in \Sigma\}$. Let $\Sigma_{1} \subset \partial B_{1}$ be an open set of $\partial B_{1}$ such that $\bar{\Sigma} \subset \Sigma_{1}$ and $\overline{\mathcal{C}}_{1} \subset \mathcal{T}_{1}\left(U \cap Q_{+}\right)$, where $\mathcal{C}_{1}=\left\{r \sigma ; r \in(0,1)\right.$ and $\left.\sigma \in \Sigma_{1}\right\}$.

Let $\mathcal{T}: \Omega \rightarrow B_{1}$ be defined by

$$
\mathcal{T}=\mathcal{T}_{1} \circ \mathcal{H}
$$

As in the proof of Lemma 4.1, set, for $x^{\prime} \in B_{1}$,

$$
f^{\prime}\left(x^{\prime}\right)=\frac{\nabla \mathcal{T}(x)}{J(x)} f(x) \text { with } x^{\prime}=\mathcal{T}(x) \text { and } J(x)=\operatorname{det} \mathcal{T}(x),
$$

and

$$
F^{\prime}\left(x^{\prime}\right)=-\int_{0}^{1} t x^{\prime} \times f^{\prime}\left(t x^{\prime}\right) d t .
$$


Define, in $\Omega$,

$$
T_{1}(f)(x)=\nabla \mathcal{T}^{T}(x) F^{\prime}\left(x^{\prime}\right) \text { with } x^{\prime}=\mathcal{T}(x) .
$$

As in the proof of Lemma 4.1, we have

$$
\nabla \times T_{1}(f)=f \text { in } \Omega
$$

and

$$
\int_{\Omega}\left|T_{1}(f)\right|^{2} d x \leqslant C \int_{\Omega} d_{\partial \Omega}^{\alpha}|f|^{2} d x
$$

Let $V$ be an open subset of $\mathbb{R}^{3}$ such that $\mathcal{H}^{-1}(\bar{S}) \subset V$ and $V \cap \Omega=\mathcal{H}^{-1}\left(\mathcal{T}^{-1}\left(\mathcal{C}_{1}\right)\right)$. Such a $V$ exists by the choice of $\mathcal{C}_{1}$. Assume that $f=0$ in $\mathcal{H}^{-1}\left(U \cap Q_{+}\right)$. Then $f^{\prime}\left(x^{\prime}\right)=0$ for $x^{\prime} \in \mathcal{C}_{1}$. It follows from (4.7) that $F^{\prime}\left(x^{\prime}\right)=0$ for $x^{\prime} \in \mathcal{C}_{1}$. This implies that $T_{1}(f)=0$ in $\mathcal{H}^{-1}\left(\mathcal{T}^{-1}\left(\mathcal{C}_{1}\right)\right)=V \cap \Omega$. The proof is complete.

In what follows, we use several times the following simple compactness result whose proof is omitted.

Lemma 4.3. Let $\Omega$ be a bounded open subset of $\mathbb{R}^{d}$ of class $C^{1}$ and let $\gamma_{1}<\gamma_{2}$. Assume that $\left(u_{n}\right) \subset L_{\mathrm{loc}}^{2}(\Omega)$ is such that $u_{n} \rightarrow u$ in $L_{\mathrm{loc}}^{2}(\Omega)$ as $n \rightarrow+\infty$ and

$$
\sup _{n}\left\|u_{n}\right\|_{L^{2}\left(d_{\partial \Omega}^{\left.\gamma_{1}, \Omega\right)}\right.}<+\infty \text {. }
$$

Then $u_{n} \rightarrow u$ in $L^{2}\left(d_{\partial \Omega}^{\gamma_{2}}, \Omega\right)$ as $n \rightarrow+\infty$.

Here and in what follows, for a non-negative, measurable function $g$ defined in $\Omega$, one denotes, for $u \in L_{\text {loc }}^{2}(\Omega)$,

$$
\|u\|_{L^{2}(g, \Omega)}=\left(\int_{\Omega} g|u|^{2}\right)^{1 / 2}
$$

For $\left(u_{n}\right) \subset L_{\text {loc }}^{2}(\Omega)$ and $u \in L_{\text {loc }}^{2}(\Omega)$, one says that $u_{n} \rightarrow u$ in $L^{2}(g, \Omega)$ as $n \rightarrow+\infty$ if $\lim _{n \rightarrow+\infty}\left\|u_{n}-u\right\|_{L^{2}(g, \Omega)}=0$.

Let $\Omega$ be a bounded open subset of $\mathbb{R}^{3}$ with Lipschitz boundary. We denote

$$
H_{0}(\operatorname{curl}, \Omega)=\{u \in H(\operatorname{curl}, \Omega) ; u \times \nu=0 \text { on } \partial \Omega\} .
$$

In the proof of Lemma 4.5, we also use the following form of Poincaré's lemma:

Lemma 4.4. Let $\Omega$ be a simply connected, bounded, open subset of $\mathbb{R}^{3}$ of class $C^{2}$. There exists a linear continuous transformation $T_{0}: H_{0}(\operatorname{curl}, \Omega) \rightarrow\left[H_{0}^{1}(\Omega)\right]^{3}$ such that

$$
\nabla \times T_{0}(u)=\nabla \times u \text { in } \Omega \text {. }
$$

Consequently, for every $s>0$, for every $0 \leqslant \alpha<2$, and for every $\beta \geqslant 0$, there exists a constant $C_{s}=C(s, \alpha, \beta, \Omega)>0$ such that

$$
\left\|T_{0}(u)\right\|_{L^{2}\left(d_{\partial \Omega}^{-\alpha} \Omega\right)} \leqslant s\|u\|_{H(\operatorname{curl}, \Omega)}+C_{s}\|u\|_{L^{2}\left(d_{\partial \Omega}^{\beta}, \Omega\right)} .
$$

Proof. We only prove (4.8) and we prove it by contradiction. Assertion (4.4) is known, see e.g. [12, Theorem 3.6], [10, Theorem 8.16]. Assume that (4.8) does not hold. There exists a sequence of $\left(u_{n}\right) \subset H_{0}(\operatorname{curl}, \Omega)$ such that

$$
1=\left\|T_{0}\left(u_{n}\right)\right\|_{L^{2}\left(d_{\partial \Omega}^{-\alpha}, \Omega\right)} \geqslant s\left\|u_{n}\right\|_{H(\operatorname{curl}, \Omega)}^{2}+n\left\|u_{n}\right\|_{L^{2}\left(d_{\partial \Omega}^{\beta}, \Omega\right)}^{2} .
$$

Since $T_{0}$ is continuous, it follows that the sequence $\left(\left\|T_{0}\left(u_{n}\right)\right\|_{H^{1}(\Omega)}\right)$ is bounded. Without loss of generality, one may assume that $T_{0}\left(u_{n}\right) \rightarrow v$ in $\left[L^{2}(\Omega)\right]^{3}$ and weakly in $\left[H^{1}(\Omega)\right]^{3}$. By Hardy's inequality

$$
\int_{\Omega} d_{\partial \Omega}^{2}|v|^{-2} \leqslant C_{\Omega} \int_{\Omega}|\nabla v|^{2}
$$


we derive that

$$
\int_{\Omega} d_{\partial \Omega}^{-2}\left|T_{0}\left(u_{n}\right)\right|^{2} \leqslant C
$$

Since $0 \leqslant \alpha<2$, by Lemma 4.3, without loss of generality, one may assume that $T_{0}\left(u_{n}\right) \rightarrow v$ in $\left[L^{2}\left(d_{\partial \Omega}^{-\alpha}, \Omega\right)\right]^{3}$.

From (4.9), one may assume as well that $u_{n} \rightarrow 0$ weakly in $H_{0}(\operatorname{curl}, \Omega)$. Since $T_{0}$ is linear and continuous, this in turn implies that $v=T_{0}\left(\lim _{n \rightarrow+\infty} u_{n}\right)=T_{0}(0)=0$. This contradicts the fact $\|v\|_{L^{2}\left(d_{\partial \Omega}^{-\alpha}, \Omega\right)}=\lim _{n \rightarrow+\infty}\left\|T_{0}\left(u_{n}\right)\right\|_{L^{2}\left(d_{\partial \Omega}^{-\alpha}, \Omega\right)}=1$. Therefore, (4.8) holds.

We now present the key ingredient of the proof of Theorem 2.2, which is a variant of Lemma 3.5.

Lemma 4.5. Let $\tau>0,0<\delta<1, s>0$, and let $\Omega$ be a connected component of $D_{-\tau}$. Let $\varepsilon, \hat{\varepsilon}, \mu, \hat{\mu}$ be real, symmetric, uniformly elliptic matrix-valued functions defined in $\Omega$. Let $J_{e}, J_{m}, \hat{J}_{e}, \hat{J}_{m} \in\left[L^{2}(\Omega)\right]^{3}$ and $(E, H),(\hat{E}, \hat{H}) \in[H(\operatorname{curl}, \Omega)]^{2}$ be such that

$$
\left\{\begin{array} { c l } 
{ \nabla \times E = i \omega \mu H + J _ { e } } & { \text { in } \Omega , } \\
{ \nabla \times H = - i \omega \varepsilon E + J _ { m } } & { \text { in } \Omega , }
\end{array} \quad \left\{\begin{array}{cl}
\nabla \times \hat{E}=i \omega \hat{\mu} \hat{H}+\hat{J}_{e} & \text { in } \Omega, \\
\nabla \times \hat{H}=-i \omega \hat{\varepsilon} \hat{E}+\hat{J}_{m} & \text { in } \Omega,
\end{array}\right.\right.
$$

and

$$
E \times \nu=\hat{E} \times \nu \quad \text { and } \quad H \times \nu=\hat{H} \times \nu \text { on } \partial \Omega \cap \Gamma .
$$

We have

1) if, for some $c>0$ and $0 \leqslant \alpha_{1}<2$,

$$
\hat{\varepsilon}-\varepsilon \geqslant c d_{\Gamma}^{\alpha_{1}} I \text { in } \Omega \quad \text { or } \quad \varepsilon-\hat{\varepsilon} \geqslant c d_{\Gamma}^{\alpha_{1}} I \text { in } \Omega
$$

then, for every $s>0$ and for every $0 \leqslant \beta<2$, there exists a constant $C_{s}>0$, depending only on $s, \beta, c, \alpha_{1}, \Omega$, and the ellipticity of $\varepsilon, \hat{\varepsilon}, \mu, \hat{\mu}$ such that

$$
\begin{aligned}
\int_{\Omega}|\langle(\varepsilon-\hat{\varepsilon}) E, E\rangle|+|E-\hat{E}|^{2} \leqslant s \int_{\Omega}|\mu H-\hat{\mu} \hat{H}|^{2}+C_{s} \int_{\Omega}\left|J_{m}-\hat{J}_{m}\right||\hat{E}| \\
+C_{s} \int_{\Omega} d_{\Gamma}^{\beta}|(E, \hat{E}, H, \hat{H})|^{2}+\left|\left(J_{e}, \hat{J}_{e}, J_{m}, \hat{J}_{m}\right)\right|^{2}
\end{aligned}
$$

2) if, for some $c>0$ and $0 \leqslant \alpha_{2}<2$,

$$
\hat{\mu}-\mu \geqslant c d_{\Gamma}^{\alpha_{1}} I \text { in } \Omega \quad \text { or } \quad \mu-\hat{\mu} \geqslant c d_{\Gamma}^{\alpha_{1}} I \text { in } \Omega
$$

then, for every $s>0$ and for every $0 \leqslant \beta<2$, there exists a constant $C_{s}>0$, depending only on $s, \beta, c, \alpha_{2}, \Omega$, and the ellipticity of $\varepsilon, \hat{\varepsilon}, \mu, \hat{\mu}$ such that

$$
\begin{aligned}
\int_{\Omega}|\langle(\mu-\hat{\mu}) H, H\rangle|+|H-\hat{H}|^{2} \leqslant s & \int_{\Omega}|\varepsilon E-\hat{\varepsilon} \hat{E}|^{2}+C_{s} \int_{\Omega}\left|J_{e}-\hat{J}_{e}\right||\hat{H}| \\
& +C_{s} \int_{\Omega} d_{\Gamma}^{\beta}|(E, \hat{E}, H, \hat{H})|^{2}+\left|\left(J_{e}, \hat{J}_{e}, J_{m}, \hat{J}_{m}\right)\right|^{2} .
\end{aligned}
$$

Proof. We only establish assertion 1) and assume that $\varepsilon-\hat{\varepsilon} \geqslant c d_{\Gamma}^{\alpha_{1}} I$. The proof of assertion 1 ) in the case $\hat{\varepsilon}-\varepsilon \geqslant c d_{\Gamma}^{\alpha_{1}} I$ and the proof of assertion 2) can be derived similarly. Without loss of generality, one may assume that $s$ is small. 
We use local charts for $\partial \Omega \cap \Gamma$. Let $\ell \geqslant 1$ and let $\varphi_{k} \in C_{c}^{1}\left(\mathbb{R}^{3}\right), U_{k} \subset \mathbb{R}^{3}$ open ball, and $\mathcal{H}_{k}: U_{k} \rightarrow Q$ for $1 \leqslant k \leqslant \ell$ be such that $\mathcal{H}_{k}$ is a diffeomorphism, $\mathcal{H}_{k}\left(U_{k} \cap \Omega\right)=Q_{+}$, and $\mathcal{H}_{k}\left(U_{k} \cap \Gamma\right)=Q_{0}$, supp $\varphi_{k} \Subset U_{k}$, and $\Phi=1$ in a neighborhood of $\partial \Omega \cap \Gamma$, where

$$
\Phi:=\sum_{k=1}^{\ell} \varphi_{k} \text { in } \mathbb{R}^{3} .
$$

By Lemma 4.2, for $1 \leqslant k \leqslant \ell$, there exists $\hat{\mathbf{E}}_{k} \in H\left(\operatorname{curl}, U_{k} \cap \Omega\right)$ and an open $V_{k} \subset \mathbb{R}^{3}$ containing $\overline{\partial U_{k} \cap \Omega}$ such that

$$
\begin{gathered}
\nabla \times \hat{\mathbf{E}}_{k}=\nabla \times\left(\varphi_{k} \hat{E}\right) \text { in } U_{k} \cap \Omega, \\
\left\|\hat{\mathbf{E}}_{k}\right\|_{L^{2}\left(U_{k} \cap \Omega\right)} \leqslant C\|\hat{E}\|_{L^{2}\left(d_{\Gamma}^{\beta}, U_{k} \cap \Omega\right)}, \\
\hat{\mathbf{E}}_{k}=0 \text { in } U_{k} \cap \Omega \cap V_{k} .
\end{gathered}
$$

Here and in what follows, $C$ denotes a positive constant depending only on $c, \alpha_{1}, \beta, \Omega, \varepsilon, \hat{\varepsilon}, \mu$, and $\hat{\mu}$; thus $C$ is independent of $s$.

Set, for $1 \leqslant k \leqslant \ell$,

$$
\delta_{\varphi_{k} E}=\varphi_{k}(E-\hat{E}) \text { in } U_{k} \cap \Omega
$$

Let $s_{1}$ be a small positive number defined later. Since $(E-\hat{E}) \times \nu=0$ on $\partial \Omega \cap \Gamma$, by Lemma 4.4, for $1 \leqslant k \leqslant \ell$, there exists $\delta_{\mathbf{E}_{k}} \in H_{0}^{1}\left(U_{k} \cap \Omega\right)$ such that

$$
\begin{gathered}
\nabla \times \delta_{\mathbf{E}_{k}}=\nabla \times \delta_{\varphi_{k} E} \text { in } U_{k} \cap \Omega \\
\left\|\delta_{\mathbf{E}_{k}}\right\|_{L^{2}\left(d_{\partial \Omega}^{-\beta}, U_{k} \cap \Omega\right)} \leqslant C_{s_{1}}\left\|\delta_{\varphi_{k} E}\right\|_{L^{2}\left(d_{\Gamma}^{\beta}, U_{k} \cap \Omega\right)}+s_{1}\left\|\delta_{\varphi_{k} E}\right\|_{H\left(\operatorname{curl}, U_{k} \cap \Omega\right)} .
\end{gathered}
$$

It is clear then

$$
\delta_{\mathbf{E}_{k}}=0 \text { in } \partial\left(U_{k} \cap \Omega\right)
$$

We have, in $\Omega$,

$$
\begin{aligned}
\nabla \times\left(\varphi_{k} E-\varphi_{k} \hat{E}\right) & =\nabla \varphi_{k} \times(E-\hat{E})+\varphi_{k} \nabla(E-\hat{E}) \\
& \stackrel{(4.10)}{=} \nabla \varphi_{k} \times(E-\hat{E})+i \omega \varphi_{k}(\mu H-\hat{\mu} \hat{H})+\varphi_{k}\left(J_{e}-\hat{J}_{e}\right) .
\end{aligned}
$$

We derive from (4.18) that

$$
\left\|\delta_{\mathbf{E}_{k}}\right\|_{L^{2}\left(d_{\partial \Omega}^{-\beta} U_{k} \cap \Omega\right)} \leqslant C_{s_{1}}\|E-\hat{E}\|_{L^{2}\left(d_{\Gamma}^{\beta}, U_{k} \cap \Omega\right)}+C s_{1}\left\|\left(E-\hat{E}, \mu H-\hat{\mu} \hat{H}, J_{e}-\hat{J}_{e}\right)\right\|_{L^{2}\left(U_{k} \cap \Omega\right)} .
$$

For $1 \leqslant k \leqslant \ell$, let $\xi_{k}, \eta_{k} \in H^{1}\left(U_{k} \cap \Omega\right)$ be such that

$$
\nabla \xi_{k}=\delta_{\varphi_{k} E}-\delta_{\mathbf{E}_{k}} \quad \text { and } \quad \nabla \eta_{k}=\varphi_{k} \hat{E}-\hat{\mathbf{E}}_{k} \text { in } U_{k} \cap \Omega
$$

Such $\xi_{k}$ and $\eta_{k}$ exist by Poincare's lemma, (4.14), and (4.17); moreover, one can assume that

$$
\xi_{k}=0 \text { on } \partial\left(U_{k} \cap \Omega\right) \quad \text { and } \quad \eta_{k}=0 \quad \text { on } \partial U_{k} \cap \Omega
$$

since $\nabla \xi_{k} \times \nu=0$ on $\partial\left(U_{k} \cap \Omega\right)$ by (4.19) and $\nabla \eta_{k} \times \nu=0$ on $\partial U_{k} \cap \Omega$ by (4.16).

Extend $\xi_{k}$ and $\eta_{k}$ by 0 in $\Omega \backslash U_{k}$ and still denote these extensions by $\xi_{k}$ and $\eta_{k}$. Set, in $\Omega$,

$$
\begin{gathered}
F_{E}=\Phi\left(\varepsilon E-\hat{\varepsilon} \hat{E}+\frac{i}{\omega} J_{m}-\frac{i}{\omega} \hat{J}_{m}\right)+\frac{i}{\omega} \nabla \Phi \times(H-\hat{H}), \\
\delta_{f}=\sum_{k=1}^{\ell} \delta_{\mathbf{E}_{k}}, \quad f=\sum_{k=1}^{\ell} \hat{\mathbf{E}}_{k}, \quad \text { and } \quad \delta_{\Phi E}=\Phi(E-\hat{E}) .
\end{gathered}
$$


It follows from (4.21) and (4.24) that, in $\Omega$,

$$
\sum_{k=1}^{\ell} \nabla \xi_{k}=\delta_{\Phi E}-\delta_{f} \quad \text { and } \quad \sum_{k=1}^{\ell} \nabla \eta_{k}=\Phi \hat{E}-f
$$

and from (4.15) and (4.20) that

$$
\begin{aligned}
&\left\|\delta_{f}\right\|_{L^{2}\left(d_{\partial \Omega}^{-\beta}, \Omega\right)}+\|f\|_{L^{2}(\Omega)} \leqslant C_{s_{1}}\left\|\left(J_{e}, \hat{J}_{e}\right)\right\|_{L^{2}(\Omega)} \\
&+C_{s_{1}}\|(E, \hat{E}, H, \hat{H})\|_{L^{2}\left(d_{\Gamma}^{\beta}, \Omega\right)}+C s_{1}\|(E-\hat{E}, \mu H-\hat{\mu} \hat{H})\|_{L^{2}\left(U_{k} \cap \Omega\right)} .
\end{aligned}
$$

Since, by (4.10) and (4.23),

$$
F_{E}=\frac{i}{\omega} \nabla \times(\Phi H)-\frac{i}{\omega} \nabla \times(\Phi \hat{H}) \text { in } \Omega
$$

and, by (4.11),

$$
(\nabla \times(\Phi H-\Phi \hat{H})) \cdot \nu=\operatorname{div}_{\Gamma}((\Phi H-\Phi \hat{H}) \times \nu)=0 \text { on } \partial \Omega \cap \Gamma,
$$

we obtain

$$
\operatorname{div} F_{E}=0 \text { in } \Omega \quad \text { and } \quad F_{E} \cdot \nu=0 \text { on } \partial \Omega \cap \Gamma .
$$

From (4.22) and (4.27), we have, for $1 \leqslant k \leqslant \ell$,

$$
\int_{\Omega}\left\langle F_{E}, \nabla \eta_{k}\right\rangle=0
$$

Summing this identity with respect to $k$ and using (4.25), we obtain

$$
\int_{\Omega}\left\langle F_{E}, \Phi \hat{E}\right\rangle=\int_{\Omega}\left\langle F_{E}, f\right\rangle .
$$

We have, for $1 \leqslant k \leqslant \ell$,

$$
\frac{i}{\omega}\left(\nabla \times\left(\varphi_{k} \hat{H}\right)-\nabla \varphi_{k} \times \hat{H}\right)=\frac{i}{\omega} \varphi_{k} \nabla \times \hat{H} \stackrel{(4.10)}{=} \hat{\varepsilon}\left(\varphi_{k} \hat{E}\right)+\frac{i}{\omega} \varphi_{k} \hat{J}_{m} \text { in } \Omega .
$$

It follows that

$$
\operatorname{div}\left[\hat{\varepsilon}\left(\varphi_{k} \hat{E}\right)\right]=-\frac{i}{\omega} \operatorname{div}\left(\varphi_{k} \hat{J}_{m}+\nabla \varphi_{k} \times \hat{H}\right) \text { in } \Omega .
$$

We derive that, for $1 \leqslant k, l \leqslant \ell$,

$$
\int_{\Omega}\left\langle\hat{\varepsilon} \nabla \xi_{l}, \varphi_{k} \hat{E}\right\rangle=\frac{i}{\omega} \int_{\Omega}\left\langle\nabla \xi_{l}, \varphi_{k} \hat{J}_{m}+\nabla \varphi_{k} \times \hat{H}\right\rangle,
$$

since $\xi_{l}=0$ on $\partial \Omega$. Summing with respect to $k$ and $l$ and using (4.25), we get

$$
\int_{\Omega}\left\langle\hat{\varepsilon}\left(\delta_{\Phi E}-\delta_{f}\right), \Phi \hat{E}\right\rangle=\frac{i}{\omega} \int_{\Omega}\left\langle\delta_{\Phi E}-\delta_{f}, \Phi \hat{J}_{m}+\nabla \Phi \times \hat{H}\right\rangle .
$$

This yields

$$
\int_{\Omega}\left\langle\hat{\varepsilon} \delta_{\Phi E}, \Phi \hat{E}\right\rangle=\int_{\Omega}\left\langle\hat{\varepsilon} \delta_{f}, \Phi \hat{E}\right\rangle+\frac{i}{\omega} \int_{\Omega}\left\langle\delta_{\Phi E}-\delta_{f}, \Phi \hat{J}_{m}+\nabla \Phi \times H\right\rangle .
$$

Noting that $\varepsilon E-\hat{\varepsilon} \hat{E}=\varepsilon(E-\hat{E})+(\varepsilon-\hat{\varepsilon}) \hat{E}$ in $\Omega$, we obtain from (4.23) that

$$
F_{E}=\varepsilon \delta_{\Phi E}+(\varepsilon-\hat{\varepsilon}) \Phi \hat{E}+\frac{i}{\omega} \Phi\left(J_{m}-\hat{J}_{m}\right)+\frac{i}{\omega} \nabla \Phi \times(H-\hat{H}) \text { in } \Omega .
$$


Subtracting (4.30) from (4.28) and using (4.31), we have

$$
\begin{aligned}
\int_{\Omega}\left\langle(\varepsilon-\hat{\varepsilon}) \delta_{\Phi E}, \Phi \hat{E}\right\rangle & +\int_{\Omega}\langle(\varepsilon-\hat{\varepsilon}) \Phi \hat{E}, \Phi \hat{E}\rangle \\
=- & \frac{i}{\omega} \int_{\Omega}\left\langle\Phi\left(J_{m}-\hat{J}_{m}\right)+\nabla \Phi \times(H-\hat{H}), \Phi \hat{E}\right\rangle \\
& \quad+\int_{\Omega}\left\langle F_{E}, f\right\rangle-\int_{\Omega}\left\langle\hat{\varepsilon} \delta_{f}, \Phi \hat{E}\right\rangle-\frac{i}{\omega} \int_{\Omega}\left\langle\delta_{\Phi E}-\delta_{f}, \Phi \hat{J}_{m}+\nabla \Phi \times H\right\rangle .
\end{aligned}
$$

From (4.22) and (4.27), we derive that, for $1 \leqslant k \leqslant \ell$,

$$
\int_{\Omega}\left\langle F_{E}, \nabla \xi_{k}\right\rangle=0
$$

Summing (4.33) with respect to $k$ and using (4.25), we get

$$
\int_{\Omega}\left\langle F_{E}, \delta_{\Phi E}-\delta_{f}\right\rangle=0
$$

It follows from (4.31) that

$$
\begin{aligned}
\int_{\Omega}\left\langle\varepsilon \delta_{\Phi E}, \delta_{\Phi E}\right\rangle+\int_{\Omega}\langle & {\left.[\varepsilon-\hat{\varepsilon}] \Phi \hat{E}, \delta_{\Phi E}\right\rangle } \\
& =-\frac{i}{\omega} \int_{\Omega}\left\langle\Phi\left(J_{m}-\hat{J}_{m}\right)+\nabla \Phi \times(H-\hat{H}), \delta_{\Phi E}\right\rangle+\int_{\Omega}\left\langle F_{E}, \delta_{f}\right\rangle .
\end{aligned}
$$

We have, by (4.31),

$$
\begin{aligned}
\left|\int_{\Omega}\left\langle F_{E}, f\right\rangle\right| \leqslant C\left(\int_{\Omega}|f|^{2}\right)^{1 / 2}\left(\int_{\Omega}|\langle(\varepsilon-\hat{\varepsilon}) \Phi \hat{E}, \Phi \hat{E}\rangle|+\left|\delta_{\Phi E}\right|^{2}\right)^{1 / 2} & \\
& +C \int_{\Omega}\left|\left(f, J_{m}, \hat{J}_{m}, \nabla \Phi \times H, \nabla \Phi \times \hat{H}\right)\right|^{2} .
\end{aligned}
$$

Since $\varepsilon-\hat{\varepsilon} \geqslant 0$ and $\varepsilon$ is uniformly elliptic, we deduce that

$$
\langle(\varepsilon-\hat{\varepsilon}) x, x\rangle+\langle\varepsilon y, y\rangle+\langle(\varepsilon-\hat{\varepsilon}) x, y\rangle+\langle(\varepsilon-\hat{\varepsilon}) y, x\rangle \geqslant C(\langle(\varepsilon-\hat{\varepsilon}) x, x\rangle+\langle\varepsilon y, y\rangle),
$$

for any $x, y \in \mathbb{R}^{3}$. It follows from (4.32), (4.34), and (4.35) that

$$
\begin{aligned}
& \int_{\Omega}|\langle(\varepsilon-\hat{\varepsilon}) \Phi \hat{E}, \Phi \hat{E}\rangle|+\left|\delta_{\varphi E}\right|^{2} \\
& \leqslant C \int_{\Omega}\left|\left(J_{m}, \hat{J}_{m}, \nabla \Phi \times H, \nabla \Phi \times \hat{H}, f, \delta_{f}\right)\right|^{2}+C\left|\int_{\Omega}\left\langle\hat{\varepsilon} \delta_{f}, \Phi E\right\rangle\right| \\
& \quad+C \int_{\Omega}\left|J_{m}-\hat{J}_{m}\right||\hat{E}|+C \int_{\Omega}|\nabla \Phi \times(H-\hat{H})||\Phi \hat{E}| .
\end{aligned}
$$

We next estimate $\left|\int_{\Omega}\left\langle\hat{\varepsilon} \delta_{f}, \Phi E\right\rangle\right|$. Since

$$
\begin{aligned}
\left|\int_{\Omega}\left\langle\hat{\varepsilon} \delta_{f}, \Phi E\right\rangle\right| & =\left|\int_{\Omega}\left\langle(\varepsilon-\hat{\varepsilon})^{-1 / 2} \hat{\varepsilon} \delta_{f},(\varepsilon-\hat{\varepsilon})^{1 / 2} \Phi E\right\rangle\right| \\
& \leqslant \frac{1}{4 s} \int_{\Omega}\left\langle(\varepsilon-\hat{\varepsilon})^{-1 / 2} \hat{\varepsilon} \delta_{f},(\varepsilon-\hat{\varepsilon})^{-1 / 2} \hat{\varepsilon} \delta_{f}\right\rangle+s \int_{\Omega}\left\langle(\varepsilon-\hat{\varepsilon})^{1 / 2} \Phi E,(\varepsilon-\hat{\varepsilon})^{1 / 2} \Phi E\right\rangle
\end{aligned}
$$


and $\varepsilon-\hat{\varepsilon} \geqslant c d_{\Gamma}^{\alpha_{1}} I$ in $\Omega$, we obtain

$$
\left|\int_{\Omega}\left\langle\hat{\varepsilon} \delta_{f}, \Phi E\right\rangle\right| \leqslant \frac{C}{s} \int_{\Omega} d_{\Gamma}^{-2 \alpha_{1}}\left|\delta_{f}\right|^{2}+s \int_{\Omega}\langle(\varepsilon-\hat{\varepsilon}) \Phi E, \Phi E\rangle .
$$

Combining (4.26) and (4.37) yields

$$
\begin{aligned}
\left|\int_{\Omega}\left\langle\hat{\varepsilon} \delta_{f}, \Phi E\right\rangle\right| \leqslant C_{s, s_{1}} \int_{\Omega}\left|\left(J_{e}, \hat{J}_{e}\right)\right|^{2}+C_{s, s_{1}} \int_{\Omega} d_{\Gamma}^{\beta}|(E, \hat{E}, H, \hat{H})|^{2} & \\
& +\frac{C s_{1}}{s} \int_{\Omega}|(\mu H-\hat{\mu} \hat{H}, E-\hat{E})|^{2}+s \int_{\Omega}\langle(\varepsilon-\hat{\varepsilon}) \Phi E, \Phi E\rangle .
\end{aligned}
$$

Using (4.26) and the fact $\Phi=1$ in a neighborhood of $\Gamma \cap \partial \Omega$, we derive from (4.36) and (4.38) that

$$
\begin{aligned}
& \int_{\Omega}|\langle(\varepsilon-\hat{\varepsilon}) \Phi \hat{E}, \Phi \hat{E}\rangle|+\left|\delta_{\varphi E}\right|^{2} \\
& \leqslant C_{s, s_{1}} \int_{\Omega}\left|\left(J_{e}, \hat{J}_{e}, J_{m}, \hat{J}_{m}\right)\right|^{2}+C_{s, s_{1}} \int_{\Omega} d_{\Gamma}^{\beta}|(E, \hat{E}, H, \hat{H})|^{2} \\
& +\frac{C s_{1}}{s} \int_{\Omega}|(\mu H-\hat{\mu} \hat{H}, E-\hat{E})|^{2} \\
& \quad+C s \int_{\Omega}\langle(\varepsilon-\hat{\varepsilon}) \Phi E, \Phi E\rangle+C \int_{\Omega}\left|J_{m}-\hat{J}_{m}\right||\hat{E}| .
\end{aligned}
$$

Take $s_{1}=C s^{2}$. One derives from (4.39) that, for $s$ small,

$$
\begin{aligned}
\int_{\Omega}|\langle(\varepsilon-\hat{\varepsilon}) \Phi \hat{E}, \Phi \hat{E}\rangle|+\left|\delta_{\varphi E}\right|^{2} \leqslant C_{s} \int_{\Omega}\left|\left(J_{e}, \hat{J}_{e}, J_{m}, \hat{J}_{m}\right)\right|^{2}+C_{s} \int_{\Omega} d_{\Gamma}^{\beta}|(E, \hat{E}, H, \hat{H})|^{2} \\
+s \int_{\Omega}|(\mu H-\hat{\mu} \hat{H})|^{2}+C \int_{\Omega}\left|J_{e}-\hat{J}_{e}\right||\hat{H}|,
\end{aligned}
$$

which implies (4.12).

Remark 4.2. The proof of Lemma 4.5 involves local charts. The involvement is of a global character in the sense that one has to combine local charts before deriving desired estimates in some parts of the proof, see (4.36) and (4.39). In fact, one cannot derive variants of (4.12) and (4.13) for $\left(\varphi_{k} E, \varphi_{k} \hat{E}, \varphi_{k} H, \varphi_{k} \hat{H}\right)$. To this end, note that, in $U_{k} \cap \Omega$,

$$
\left\{\begin{array} { c } 
{ \nabla \times ( \varphi _ { k } E ) = i \omega \mu ( \varphi _ { k } H ) + J _ { e , k } , } \\
{ \nabla \times ( \varphi _ { k } H ) = - i \omega \varepsilon ( \varphi _ { k } E ) + J _ { m , k } , }
\end{array} \quad \left\{\begin{array}{c}
\nabla \times\left(\varphi_{k} \hat{E}\right)=i \omega \hat{\mu}\left(\varphi_{k} \hat{H}\right)+\hat{J}_{e, k} \\
\nabla \times\left(\varphi_{k} \hat{H}\right)=-i \omega \hat{\varepsilon}\left(\varphi_{k} \hat{E}\right)+\hat{J}_{m, k},
\end{array}\right.\right.
$$

where

$$
\begin{array}{cl}
J_{e, k}=\varphi_{k} J_{e}+\nabla \varphi_{k} \times E, & \hat{J}_{e, k}=\varphi_{k} \hat{J}_{e}+\nabla \varphi_{k} \times \hat{E}, \\
J_{m, k}=\varphi_{k} \hat{J}_{m}+\nabla \varphi_{k} \times H, & \hat{J}_{m, k}=\varphi_{k} \hat{J}_{m}+\nabla \varphi_{k} \times \hat{H} .
\end{array}
$$

Due to the terms $\nabla \varphi_{k} \times E, \nabla \varphi_{k} \times \hat{E}, \nabla \varphi_{k} \times H$, and $\nabla \varphi_{k} \times \hat{H}$ in $J_{e, k}, \hat{J}_{e, k}, J_{m, k}$, and $\hat{J}_{m, k}$, respectively, we are not able to derive the variants of (4.12) and (4.13) for $\left(\varphi_{k} E, \varphi_{k} \hat{E}, \varphi_{k} H, \varphi_{k} \hat{H}\right)$. This combination is the key difference between the proof strategies of Lemmas 4.5] and 3.5 and makes the proof of Lemma 4.5 more involved. Another difference between the proofs is that one considers the extensions of $\varphi E_{k}$ and $\varphi_{k}(E-\hat{E})$ in the proof Lemma 4.5 instead of 
the extensions of $\varphi E_{k}$ and $\varphi_{k} \hat{E}$ as in the proof Lemma 3.5 to ensure integration by parts arguments, see (4.29) where $\xi_{k}=0$ on $\partial\left(\Omega \cap U_{k}\right)$ is required.

4.2. Proof of Theorem 2.2. For a simpler presentation, we will assume that $D_{-\tau}$ is connected. We first consider the case where $\alpha_{1}+\alpha_{2}>0$. Set

$$
\beta=\left(\max \left\{\alpha_{1}, \alpha_{2}\right\}+2\right) / 2
$$

and

$$
\delta_{E_{\delta}}=E_{\delta}-\hat{E}_{\delta} \text { in } D_{-\tau} \quad \text { and } \quad \delta_{H_{\delta}}=H_{\delta}-\hat{H}_{\delta} \text { in } D_{-\tau} .
$$

Then

$$
\max \left\{\alpha_{1}, \alpha_{2}\right\}<\beta<2 .
$$

We first prove by contradiction that

$$
\left\|\left(E_{\delta}, H_{\delta}\right)\right\|_{L^{2}\left(d_{\Gamma}^{\beta}, B_{R_{0}}\right)} \leqslant C\|J\|_{L^{2}\left(\mathbb{R}^{3}\right)} .
$$

Assume, for some $\delta_{n} \rightarrow 0$ and $J_{n} \in\left[L^{2}\left(\mathbb{R}^{3}\right)\right]^{3}$ with supp $J_{n} \subset B_{R_{0}} \backslash\left(\left(D_{-\tau} \cup \mathcal{F}^{-1}\left(D_{-\tau}\right)\right)=\varnothing\right.$ that

$$
\lim _{n \rightarrow+\infty}\left\|J_{n}\right\|_{L^{2}\left(\mathbb{R}^{3}\right)}=0 \quad \text { and } \quad\left\|\left(E_{n}, H_{n}\right)\right\|_{L^{2}\left(d_{\Gamma}^{\beta}, B_{R_{0}}\right)}=1,
$$

where $\left(E_{n}, H_{n}\right)$ is the solution corresponding to $\delta_{n}$ and $J_{n}$. Integrating by parts and using the radiating condition, as usual, we obtain

$$
\left|\Im \int_{B_{R_{0}}}\left\langle\mu_{\delta_{n}}^{-1} \nabla \times E_{n}, \nabla \times E_{n}\right\rangle-\omega^{2}\left\langle\varepsilon_{\delta_{n}} E_{n}, E_{n}\right\rangle\right| \leqslant\left|\int_{B_{R_{0}}}\left\langle i \omega J_{n}, E_{n}\right\rangle\right| \rightarrow 0 \text { as } n \rightarrow+\infty .
$$

This implies

$$
\int_{D} \delta_{n}\left(\left|E_{n}\right|^{2}+\left|H_{n}\right|^{2}\right) \rightarrow 0 \text { as } n \rightarrow+\infty
$$

By a change of variables for the Maxwell equations, see e.g. [25, Lemma 7], we have

$$
\left\{\begin{array} { c l } 
{ \nabla \times E _ { n } = i \omega \mu H _ { n } } & { \text { in } D _ { - \tau } , } \\
{ \nabla \times H _ { n } = - i \omega \varepsilon E _ { n } } & { \text { in } D _ { - \tau } , }
\end{array} \quad \left\{\begin{array}{cl}
\nabla \times \hat{E}_{n}=i \omega \hat{\mu} \hat{H}_{n}+\hat{J}_{e, n} & \text { in } D_{-\tau}, \\
\nabla \times \hat{H}_{n}=-i \omega \hat{\varepsilon} \hat{E}_{n}+\hat{J}_{m, n} & \text { in } D_{-\tau},
\end{array}\right.\right.
$$

and

$$
E_{n} \times \nu=\hat{E}_{n} \times \nu \quad \text { and } \quad H_{n} \times \nu=\hat{H}_{n} \times \nu \quad \text { on } \Gamma \text {. }
$$

Here

$$
\hat{J}_{e, n}=-\delta \omega \mathcal{F}_{*} I \hat{H}_{n} \quad \text { and } \quad \hat{J}_{m, n}=\delta \omega \mathcal{F}_{*} I \hat{E}_{n} \quad \text { in } D_{-\tau} .
$$

Note that, in $D_{-\tau}$,

and

$$
\varepsilon E-\hat{\varepsilon} \hat{E}=(\varepsilon-\hat{\varepsilon}) E+\hat{\varepsilon}(E-\hat{E})
$$

$$
\mu H-\hat{\mu} \hat{H}=(\mu-\hat{\mu}) H+\hat{\mu}(H-\hat{H}) .
$$

Applying (4.12) and (4.13) for a sufficiently small $s$ and using (4.43), we have

$$
\int_{D_{-\tau}}\left|\left\langle(\varepsilon-\hat{\varepsilon}) E_{n}, E_{n}\right\rangle\right|+\left.\int_{D_{-\tau}}\left|\left\langle(\mu-\hat{\mu}) H_{n}, H_{n}\right\rangle+\int_{D_{-\tau}}\right|\left(\delta_{E_{n}}, \delta_{H_{n}}\right)\right|^{2} \leqslant C .
$$

This implies

$$
\left\|E_{n}\right\|_{L^{2}\left(d_{\Gamma}^{\alpha_{1}}, B_{R_{0}}\right)}+\left\|H_{n}\right\|_{L^{2}\left(d_{\Gamma}^{\alpha_{2}}, B_{R_{0}}\right)} \leqslant C .
$$

Fix $\psi \in C_{c}^{1}\left(\mathbb{R}^{3}\right)$ (arbitrary) such that $\psi=0$ in a neighborhood of $\Gamma$. We have, in $\mathbb{R}^{3}$,

$$
\nabla \times\left(\psi E_{n}\right)=\psi \nabla \times E_{n}+\nabla \psi \times E_{n} \quad \text { and } \quad \operatorname{div}\left[\varepsilon_{0}\left(\psi E_{n}\right)\right]=\psi \operatorname{div}\left(\varepsilon_{0} E_{n}\right)+\nabla \psi \cdot \varepsilon_{0} E_{n} .
$$


Using (4.40) and (4.41), and applying [25, Lemma 1], one may assume that

$$
\left(\psi E_{n}\right) \text { converges in }\left[L^{2}\left(\mathbb{R}^{3}\right)\right]^{3},
$$

which yields, since $\psi$ is arbitrary,

$$
\left(E_{n}\right) \text { converges in }\left[L_{\mathrm{loc}}^{2}\left(\mathbb{R}^{3} \backslash \Gamma\right)\right]^{3} .
$$

Similarly, one may also assume that

$$
\left(H_{n}\right) \text { converges in }\left[L_{\mathrm{loc}}^{2}\left(\mathbb{R}^{3} \backslash \Gamma\right)\right]^{3} \text {. }
$$

Moreover, from (4.45), (4.46), (4.47) and the fact $\beta>\max \left\{\alpha_{1}, \alpha_{2}\right\}$, by Lemma 4.3, one may assume that

$$
\left(\left(E_{n}, H_{n}\right)\right) \text { converges in }\left[L^{2}\left(d_{\Gamma}^{\beta}, B_{R_{0}}\right)\right]^{6}
$$

and, by (4.44),

$$
\left(\left(\delta_{E_{n}}, \delta_{H_{n}}\right)\right) \text { converges weakly in }\left[L^{2}\left(D_{-\tau}\right)\right]^{6} .
$$

Let $(E, H)$ be the limit of $\left(\left(E_{n}, H_{n}\right)\right)$ in $\left[L_{\text {loc }}^{2}\left(\mathbb{R}^{3} \backslash \Gamma\right)\right]^{6}$. From (4.44), we derive that $\left(\delta_{E}, \delta_{H}\right) \in$ $\left[L^{2}\left(D_{-\tau}\right)\right]^{2}$. From the equations of $(E, H)$, it follows that $\left(\delta_{E}, \delta_{H}\right) \in\left[H\left(\operatorname{curl}, D_{-\tau}\right)\right]^{2}$. One also has

$$
\delta_{E} \times \nu=\delta_{H} \times \nu=0 \text { on } \Gamma \text {. }
$$

We have, for $R>R_{0}$,

$$
\left|\Re \int_{\partial B_{R}} H \times \nu \cdot \bar{E}\right|=\lim _{n \rightarrow+\infty}\left|\Re \int_{\partial B_{R}} H_{n} \times \nu \cdot \bar{E}_{n}\right| \stackrel{(4.43)}{\leqslant} \limsup _{n \rightarrow+\infty}\left|\int_{B_{R}} J_{n} \bar{E}_{n}\right| \stackrel{(4.42)}{=} 0 .
$$

Since $(E, H)$ satisfies the radiating condition, it follows that

$$
E=H=0 \text { in the unbounded connected component of } \mathbb{R}^{3} \backslash \bar{D},
$$

which in turn implies, by (4.50) and the unique continuation principle, that

$$
E=H=0 \text { in } \mathbb{R}^{3} \text {. }
$$

Combining (4.42), (4.48), (4.49), and (4.51) yields a contradiction. Hence (4.41) is proved. Applying Lemma 4.5 again, we derive (2.3) from (4.41).

By the same method, one also obtains the following fact: for any $\left(\delta_{n}\right) \rightarrow 0$, up to a subsequence, $\left(\left(E_{\delta_{n}}, H_{\delta_{n}}\right)\right)$ converges in $\left[L_{\mathrm{loc}}^{2}\left(\mathbb{R}^{3} \backslash \Gamma\right)\right]^{6}$, and the limit is a radiating solution of the corresponding system.

We next consider the case $\alpha_{1}=\alpha_{2}=0$. We first prove by contradiction that

$$
\left\|\left(E_{\delta}, H_{\delta}\right)\right\|_{L^{2}\left(B_{R_{0}}\right)} \leqslant C\|J\|_{L^{2}\left(\mathbb{R}^{3}\right)} .
$$

Assume, for some $\delta_{n} \rightarrow 0$ and $J_{n} \in L^{2}\left(\mathbb{R}^{3}\right)$ with supp $J_{n} \subset B_{R_{0}}$ that

$$
\lim _{n \rightarrow+\infty}\left\|J_{n}\right\|_{L^{2}\left(\mathbb{R}^{3}\right)}=0 \quad \text { and } \quad\left\|\left(E_{n}, H_{n}\right)\right\|_{L^{2}\left(B_{R_{0}}\right)}=1,
$$

where $\left(E_{n}, H_{n}\right)$ is the solution corresponding to $\delta_{n}$ and $J_{n}$. By a change of variables for the Maxwell equations, see e.g. [25, Lemma 7], we have

$$
\left\{\begin{array} { c l } 
{ \nabla \times E _ { n } = i \omega \mu H _ { n } } & { \text { in } D _ { - \tau } , } \\
{ \nabla \times H _ { n } = - i \omega \varepsilon E _ { n } + J _ { n } } & { \text { in } D _ { - \tau } , }
\end{array} \quad \left\{\begin{array}{cl}
\nabla \times \hat{E}_{n}=i \omega \hat{\mu} \hat{H}_{n}+\hat{J}_{e, n} & \text { in } D_{-\tau}, \\
\nabla \times \hat{H}_{n}=-i \omega \hat{\varepsilon} \hat{E}_{n}+\hat{J}_{m, n} & \text { in } D_{-\tau},
\end{array}\right.\right.
$$

and

$$
E_{n} \times \nu=\hat{E}_{n} \times \nu \quad \text { and } \quad H_{n} \times \nu=\hat{H}_{n} \times \nu \quad \text { on } \Gamma \text {. }
$$

Here

$$
\hat{J}_{e, n}=-\delta \omega \mathcal{F}_{*} I \hat{H}_{n} \quad \text { and } \quad \hat{J}_{m, n}=\delta \omega \mathcal{F}_{*} I \hat{E}_{n}+\mathcal{F}_{*} J_{n} \quad \text { in } D_{-\tau} \text {. }
$$


Note that

$$
\int_{\Omega}\left|J_{n}-\hat{J}_{m, n}\right|\left|\hat{E}_{n}\right|+\left|\hat{J}_{e, n}\right|\left|\hat{H}_{n}\right| \leqslant\left(\int_{\Omega}\left|\left(J_{n}, \hat{J}_{m, n}, \hat{J}_{e, n}\right)\right|^{2}\right)^{1 / 2}\left(\int_{\Omega}\left|\left(\hat{E}_{n}, \hat{H}_{n}\right)\right|^{2}\right)^{1 / 2} .
$$

As in (4.45), we then have

$$
\left\|E_{n}\right\|_{L^{2}\left(B_{R_{0}}\right)}+\left\|H_{n}\right\|_{L^{2}\left(B_{R_{0}}\right)} \leqslant C .
$$

As in the proof of Theorem 2.1, one can prove that, up to a subsequence,

$$
\left(E_{n}, H_{n}\right) \text { converges in }\left[L_{\text {loc }}^{2}\left(\mathbb{R}^{3}\right)\right]^{12} \text {. }
$$

Moreover, the limit $\left(E_{0}, H_{0}\right)$ is in $\left[H_{\text {loc }}\left(\operatorname{curl}, \mathbb{R}^{3}\right)\right]^{2}$ and is a radiating solution of the equations

$$
\left\{\begin{array}{cl}
\nabla \times E_{0}=i \omega \mu_{0} H & \text { in } \mathbb{R}^{3} \\
\nabla \times H_{0}=-i \omega \varepsilon_{0} E & \text { in } \mathbb{R}^{3} .
\end{array}\right.
$$

By Lemma 3.7, we get

$$
E_{0}=H_{0}=0 \text { in } \mathbb{R}^{3} \text {. }
$$

This contradicts (4.52) and (4.53). The uniqueness of $\left(E_{0}, H_{0}\right)$ is a consequence of Lemma 3.7 and the strong convergence of $\left(E_{\delta}, H_{\delta}\right)$ to $\left(E_{0}, H_{0}\right)$ in $\left[L_{\mathrm{loc}}^{2}\left(\mathbb{R}^{3}\right)\right]^{12}$ can be derived as in the proof of Theorem 2.1 and are omitted.

4.3. Some applications of Theorem 2.2. In this section, we present two applications of Theorem 2.2. The first is a consequence of Theorem 2.2 with $\alpha_{1}=\alpha_{2}=0$. We have

Corollary 4.1. Let $0<\delta<1, J \in\left[L^{2}\left(\mathbb{R}^{3}\right)\right]^{3}$ with supp $J \subset B_{R_{0}}$, and let $\left(E_{\delta}, H_{\delta}\right) \in$ $\left[H_{\text {loc }}\left(\text { curl, } \mathbb{R}^{3}\right)\right]^{2}$ be the unique radiating solution of $(1.3)$. Assume that $D$ is of class $C^{2}$ and, for each connected component $O$ of $D_{-\tau} \cup \Gamma \cup D_{\tau}$ with $\tau>0$ small, the following four conditions hold

$$
\begin{aligned}
& \text { either }\left.\varepsilon^{+}\right|_{D_{-\tau} \cap O} \text { or }\left.\varepsilon^{-}\right|_{D_{\tau} \cap O} \text { is isotropic, } \\
& \text { either }\left.\mu^{+}\right|_{D_{-\tau} \cap O} \text { or }\left.\mu^{-}\right|_{D_{\tau} \cap O} \text { is isotropic, }
\end{aligned}
$$

$\varepsilon^{+}\left(x_{\Gamma}-t \nu\left(x_{\Gamma}\right)\right) \geqslant-\varepsilon^{-}\left(x_{\Gamma}+t \nu\left(x_{\Gamma}\right)\right)+c I \quad$ or $\quad-\varepsilon^{-}\left(x_{\Gamma}-t \nu\left(x_{\Gamma}\right)\right) \geqslant \varepsilon^{+}\left(x_{\Gamma}+t \nu\left(x_{\Gamma}\right)\right)+c I$ and

$\mu^{+}\left(x_{\Gamma}-t \nu\left(x_{\Gamma}\right)\right) \geqslant-\mu^{-}\left(x_{\Gamma}+t \nu\left(x_{\Gamma}\right)\right)+c I \quad$ or $\quad-\mu^{-}\left(x_{\Gamma}-t \nu\left(x_{\Gamma}\right)\right) \geqslant \mu^{+}\left(x_{\Gamma}+t \nu\left(x_{\Gamma}\right)\right)+c I$, for every $x_{\Gamma} \in \Gamma \cap O$ and for every $t \in(0, \tau)$, for some $c>0$. Then, for all $R>0$,

$$
\int_{B_{R}}\left|\left(E_{\delta}, H_{\delta}\right)\right|^{2} \leqslant C_{R}\|J\|_{L^{2}\left(\mathbb{R}^{3}\right)}^{2},
$$

for some positive constant $C_{R}$ independent of $\delta$ and $J$. Moreover, $\left(E_{\delta}, H_{\delta}\right)$ converges to $\left(E_{0}, H_{0}\right)$ strongly in $\left[L_{\mathrm{loc}}^{2}\left(\mathbb{R}^{3}\right)\right]^{6}$, as $\delta \rightarrow 0$, where $\left(E_{0}, H_{0}\right) \in\left[H_{\mathrm{loc}}\left(\mathrm{curl}, \mathbb{R}^{3}\right)\right]^{2}$ is the unique radiating solution of (1.3) with $\delta=0$. As a consequence,

$$
\int_{B_{R}}\left|\left(E_{0}, H_{0}\right)\right|^{2} \leqslant C_{R}\|J\|_{L^{2}\left(\mathbb{R}^{3}\right)}^{2}
$$

Remark 4.3. It is worth comparing Corollary 4.1 with Corollary 2.1, First, in Corollary 4.1, one does not require $\varepsilon^{+}, \varepsilon^{-}, \mu^{+}, \mu^{-}$to be $C^{1}$ near $\Gamma$. Second, in Corollary 2.1, one does not require any isotropy conditions on $\varepsilon^{+}, \varepsilon^{-}, \mu^{+}, \mu^{-}$. 
Proof. For $\tau>0$ sufficiently small (the smallness depends only on $D$ ), define $\mathcal{F}: D_{\tau} \cup \Gamma \cup$ $D_{-\tau} \rightarrow D_{\tau} \cup \Gamma \cup D_{-\tau}$ by

$$
\mathcal{F}\left(x_{\Gamma}+t \nu\left(x_{\Gamma}\right)\right)=x_{\Gamma}-t \nu\left(x_{\Gamma}\right) \quad \forall x_{\Gamma} \in \Gamma, t \in(-\tau, \tau)
$$

and set, in $D_{-\tau}$,

$$
(\hat{\varepsilon}, \hat{\mu})=\left(\mathcal{F}_{*} \varepsilon^{-}, \mathcal{F}_{*} \mu^{-}\right) .
$$

For the simplicity of the presentation, we assume that $D_{-\tau}$ is connected. We will only consider the case

$$
\begin{gathered}
\left.\varepsilon^{+}\right|_{D_{-\tau}} \text { is isotropic, } \\
\left.\mu^{+}\right|_{D_{-\tau}} \text { is isotropic, } \\
\varepsilon^{+}\left(x_{\Gamma}-t \nu\left(x_{\Gamma}\right)\right) \geqslant-\varepsilon^{-}\left(x_{\Gamma}+t \nu\left(x_{\Gamma}\right)\right)+c I,
\end{gathered}
$$

and

$$
\mu^{+}\left(x_{\Gamma}-t \nu\left(x_{\Gamma}\right)\right) \geqslant-\mu^{-}\left(x_{\Gamma}+t \nu\left(x_{\Gamma}\right)\right)+c I
$$

for every $x_{\Gamma} \in \Gamma \cap O$ and for every $0<t<\tau$, for some $c>0$. The other cases can be dealt similarly.

For $x^{\prime} \in \bar{D}_{-\tau}$, set $x=\mathcal{F}^{-1}\left(x^{\prime}\right)$ and $\mathcal{J}(x)=\operatorname{det} \nabla \mathcal{F}(x)$. We have, for $x \in \Gamma$,

$$
\nabla \mathcal{F}^{-1}(x) \nabla \mathcal{F}^{-T}(x)=I \text { and } \mathcal{J}(x)=-1 .
$$

Since $\varepsilon^{+}$is isotropic and $\varepsilon^{+}\left(x_{\Gamma}-t \nu\left(x_{\Gamma}\right)\right) \geqslant-\varepsilon^{-}\left(x_{\Gamma}+t \nu\left(x_{\Gamma}\right)\right)+c I$ in $D_{-\tau}$, it follows from the definition of $\hat{\varepsilon}$ that, for sufficiently small $\tau$,

$$
\varepsilon\left(x^{\prime}\right) \geqslant \hat{\varepsilon}\left(x^{\prime}\right)+c I / 2 \text { for } x^{\prime} \in D_{-\tau} .
$$

Similarly, we have, for sufficiently small $\tau$,

$$
\mu\left(x^{\prime}\right) \geqslant \hat{\mu}\left(x^{\prime}\right)+c I / 2 \text { for } x^{\prime} \in D_{-\tau} .
$$

Take $\tau$ sufficiently small such that (4.54) and (4.55) hold. Applying Theorem 2.2, we obtain the conclusion.

Here is a direct consequence of Corollary 4.1. Let $\Omega_{1}$ and $\Omega_{2}$ be open subsets of $D$ such that $\Omega_{1} \cap \Omega_{2}=\varnothing, \bar{D}=\bar{\Omega}_{1} \cup \bar{\Omega}_{2}, \partial \Omega_{1} \cap \partial D \neq \varnothing$, and $\partial \Omega_{2} \cap \partial D \neq \varnothing$. Assume that $\varepsilon^{-}=-\gamma_{1} I$ in $\Omega_{1}$ and $=-\gamma_{2} I$ in $\Omega_{2}$, and $\varepsilon^{+}=I$ in $\mathbb{R}^{3} \backslash D$ for some constants $\gamma_{1}, \gamma_{2}>0$ with $\min \left\{\gamma_{1}, \gamma_{2}\right\}>1$ or $\max \left\{\gamma_{1}, \gamma_{2}\right\}<1$. For $J \in L^{2}\left(\mathbb{R}^{3}\right)$ with supp $J \subset B_{R_{0}}$, let $\left(E_{\delta}, H_{\delta}\right) \in\left[H_{\text {loc }}\left(\operatorname{curl}, \mathbb{R}^{3}\right)\right]^{2}$ be the unique radiating solution of $(1.3)$ for $0<\delta<1$. We have $\left(E_{\delta}, H_{\delta}\right)$ is bounded in $\left[L_{\text {loc }}^{2}\left(\mathbb{R}^{3}\right)\right]^{6}$. Moreover, $\left(E_{\delta}, H_{\delta}\right)$ converges to $\left(E_{0}, H_{0}\right)$ strongly in $\left[L_{\text {loc }}^{2}\left(\mathbb{R}^{3}\right)\right]^{6}$, the unique radiating solution of (1.3) with $\delta=0$. Note that this setting is out of the scope of Corollary 2.1 for $\gamma_{1} \neq \gamma_{2}$.

Here is another consequence of Theorem 2.2 for which $\alpha_{1}=\alpha_{2}=1$. We first introduce

Definition 4.1. A connected component of $\Gamma$ is called strictly convex if it is the boundary of a strictly convex set.

We have

Corollary 4.2. Let $0<\delta<1, \tau>0, J \in\left[L^{2}\left(\mathbb{R}^{3}\right)\right]^{3}$ with supp $J \subset B_{R_{0}} \backslash\left(D_{\tau} \cup D_{-\tau}\right)$, and let $\left(E_{\delta}, H_{\delta}\right) \in\left[H_{\mathrm{loc}}\left(\operatorname{curl}, \mathbb{R}^{3}\right)\right]^{2}$ be the unique radiating solution of (1.3). Let $\mathbf{e}, \mathbf{m}$ be positive symmetric matrix-valued functions defined in $D_{-\tau} \cup \Gamma \cup D_{\tau}$ such that they are constant and isotropic on each connected component of their domain of definition. Assume that

$D$ is of class $C^{3}$, each connected component of $\Gamma$ is strictly convex,

$$
\left(\varepsilon^{+}, \mu^{+}\right)=(\mathbf{e}, \mathbf{m}) \text { in } D_{-\tau}, \quad \text { and } \quad\left(\varepsilon^{-}, \mu^{-}\right)=-(\mathbf{e}, \mathbf{m}) \text { in } D_{\tau} .
$$

Then, for $R>0$ and open $V \supset \Gamma$,

$$
\left\|\left(E_{\delta}, H_{\delta}\right)\right\|_{L^{2}\left(B_{R} \backslash V\right)} \leqslant C_{R, V}\|J\|_{L^{2}\left(\mathbb{R}^{3}\right)} \quad \forall R>0,
$$


for some positive constant $C_{R, V}$ independent of $\delta$ and $J$. Moreover, for a sequence $\left(\delta_{n}\right) \rightarrow 0$, up to a subsequence, $\left(E_{\delta_{n}}, H_{\delta_{n}}\right)$ converges to $\left(E_{0}, H_{0}\right)$ strongly in $L_{\mathrm{loc}}^{2}\left(\mathbb{R}^{3} \backslash \Gamma\right)$ as $n \rightarrow+\infty$, where $\left(E_{0}, H_{0}\right) \in\left[H_{\mathrm{loc}}\left(\mathrm{curl}, \mathbb{R}^{3} \backslash \Gamma\right)\right]^{2}$ is a radiating solution of (1.3) with $\delta=0$ and (4.56) holds with $\delta=0$.

Proof. For simple presentation, we assume that $D$ is convex. Let $\mathcal{F}$ be defined as follows:

$$
x_{\Gamma}-t \nu\left(x_{\Gamma}\right) \mapsto x_{\Gamma}+t\left[1+t c\left(x_{\Gamma}\right)\right] \nu\left(x_{\Gamma}\right),
$$

for $x_{\Gamma} \in \Gamma$ and $0<t<\tau$ (small). Here $c\left(x_{\Gamma}\right)=\beta \operatorname{trace} \Pi\left(x_{\Gamma}\right)$ where $\Pi\left(x_{\Gamma}\right)$ is the second fundamental form of $\Gamma$ at $x_{\Gamma}$ and $-1<\beta<0$. By taking $\beta$ sufficiently close to -1 , one can prove that, see [23, Proof of Corollary 3],

$$
\mathcal{F}_{*}(-I)-I \geqslant \gamma d_{\Gamma} I \text { in } D_{-\tau} .
$$

for some positive constant $\gamma$ (note that the definition of $\mathcal{F}_{*}$ in this paper is different from the one in [23] in which $|\operatorname{det}(\nabla \mathcal{F})|$ is used instead of $\operatorname{det}(\nabla \mathcal{F}))$. We are now in the range of the application of Theorem 2.2 and the conclusion follows.

Here is an immediate application of Corollary 4.2. Let $D$ be a smooth strictly convex set of class $C^{3}$ and assume that, for $\delta \geqslant 0$,

$$
\left(\varepsilon_{\delta}, \mu_{\delta}\right)=\left\{\begin{array}{cl}
I, I & \text { in } \mathbb{R}^{3} \backslash D, \\
-I+i \delta I,-I+i \delta I & \text { in } D .
\end{array}\right.
$$

Let $J \in\left[L^{2}\left(\mathbb{R}^{3}\right)\right]^{3}$ with supp $J \subset B_{R_{0}} \backslash\left(D_{\tau} \cup D_{-\tau}\right)$ and let $\left(E_{\delta}, H_{\delta}\right)$ be the unique radiating solution of (1.3) . As a consequence of Theorem 2.2 and [23, Corollary 3], one has

$$
\left\|\left(E_{\delta}, H_{\delta}\right)\right\|_{L^{2}\left(B_{R} \backslash V\right)} \leqslant C_{R, V}\|J\|_{L^{2}\left(\mathbb{R}^{3}\right)} \quad \forall R>0,
$$

for some positive constant $C_{R}$ independent of $\delta$ and $J$. Corollary 4.2 is a variant of [23, Corollary 3] for the Maxwell equations.

\section{Appendix A. Proof of Proposition 3.1}

We only consider the case $(\mu, \hat{\mu})$ does not satisfy the complementing conditions at some point $x_{0} \in \partial \Omega$. The other case can be dealt similarly. Then, from [2], there exist sequences $\left(\left(u_{n}, \hat{u}_{n}\right)\right) \subset H^{2}(\Omega),\left(\left(f_{n}, \hat{f}_{n}\right)\right) \subset L^{2}(\Omega),\left(p_{n}\right) \subset H^{\frac{3}{2}}(\partial \Omega)$ and $\left(q_{n}\right) \subset H^{\frac{1}{2}}(\partial \Omega)$ such that

$$
\begin{gathered}
\operatorname{div}\left(\mu \nabla u_{n}\right)=f_{n}, \quad \operatorname{div}\left(\hat{\mu} \nabla \hat{u}_{n}\right)=\hat{f}_{n} \quad \text { in } \Omega, \\
u_{n}-\hat{u}_{n}=p_{n} \quad \text { on } \partial \Omega, \\
\left(\mu \nabla u_{n}-\hat{\mu} \nabla \hat{u}_{n}\right) \cdot \nu=q_{n} \quad \text { on } \partial \Omega,
\end{gathered}
$$

$\left(\left\|f_{n}\right\|_{L^{2}(\Omega)}\right),\left(\left\|\hat{f}_{n}\right\|_{L^{2}(\Omega)}\right),\left(\left\|p_{n}\right\|_{H^{\frac{3}{2}(\partial \Omega)}}\right),\left(\left\|q_{n}\right\|_{H^{\frac{1}{2}(\partial \Omega)}}\right)$ and $\left(\left\|\left(u_{n}, \hat{u}_{n}\right)\right\|_{H^{1}(\Omega)}\right)$ are bounded and $\lim _{n \rightarrow+\infty}\left\|\left(u_{n}, \hat{u}_{n}\right)\right\|_{H^{2}(\Omega)}=+\infty$.

Let $\phi_{n} \in H^{2}$ and $\hat{\phi}_{n} \in H^{2}$ be the unique solution of

$$
\left\{\begin{array} { r l } 
{ \operatorname { d i v } ( \mu \nabla \phi _ { n } ) = f _ { n } } & { \text { in } \Omega , } \\
{ \phi _ { n } = p _ { n } } & { \text { on } \partial \Omega . }
\end{array} \quad \text { and } \quad \left\{\begin{array}{rl}
\operatorname{div}\left(\hat{\mu} \nabla \hat{\phi}_{n}\right)=\hat{f}_{n} & \text { in } \Omega, \\
\hat{\phi}_{n}=0 & \text { on } \partial \Omega .
\end{array}\right.\right.
$$

By the standard theory of elliptic equations, one has

$$
\left\|\phi_{n}\right\|_{H^{2}} \leqslant C\left(\left\|f_{n}\right\|_{L^{2}}+\left\|p_{n}\right\|_{H^{\frac{3}{2}}(\partial \Omega)}\right) \quad \text { and } \quad\left\|\hat{\phi}_{n}\right\|_{H^{2}} \leqslant C\left\|\hat{f}_{n}\right\|_{L^{2}},
$$


for some positive constant $C$ independent of $n$. Set

$$
H_{n}=\nabla u_{n}-\nabla \phi_{n} \quad \text { and } \quad \hat{H}_{n}=\nabla \hat{u}_{n}-\nabla \hat{\phi}_{n}, \text { in } \Omega .
$$

We have, in $\Omega$,

$$
\operatorname{curl} H_{n}=0=\operatorname{curl} \hat{H}_{n} \quad \text { and } \quad \operatorname{div}\left(\mu H_{n}\right)=0=\operatorname{div}\left(\hat{\mu} \hat{H}_{n}\right) .
$$

Since $u_{n}-\hat{u}_{n}=p_{n}=\phi_{n}$ on $\partial \Omega$, and $\hat{\varphi}_{n}=0$ on $\partial \Omega$, we deduce that

$$
\nu \times\left(H_{n}-\hat{H}_{n}\right)=0 \quad \text { on } \partial \Omega .
$$

Set

$$
E_{n}=\hat{E}_{n}=0 \text { in } \Omega
$$

and, in $\Omega$,

$$
J_{e, n}=-i \omega \mu H_{n}, \quad \hat{J}_{e, n}=-i \omega \hat{\mu} \hat{H}_{n}, \quad J_{m, n}=0, \quad \text { and } \quad \hat{J}_{m, n}=0 .
$$

One can easily check that $\left(E_{n}, \hat{E}_{n}, H_{n}, \hat{H}_{n}\right)$ and $\left(J_{e, n}, \hat{J}_{e, n}, J_{m, n}, \hat{J}_{e, n}\right)$ satisfies all the required properties.

\section{REFERENCES}

[1] Agmon, S., Douglis, A., and Nirenberg, L. Estimates near the boundary for solutions of elliptic partial differential equations satisfying general boundary conditions. I. Comm. Pure Appl. Math. 12 (1959), 623-727.

[2] S. Agmon, A. Douglis, and L. Nirenberg, Estimates near the boundary for solutions of elliptic partial differential equations satisfying general boundary conditions. II, Comm. Pure Appl. Math. 17 (1964), 35-92.

[3] H. Ammari, G. Ciraolo, H. Kang, H. Lee, and G. W. Milton, Spectral theory of a Neumann-Poincaré-type operator and analysis of cloaking due to anomalous localized resonance, Arch. Rational Mech. Anal. 218 (2013), 667-692.

[4] J. Ball, Y. Capdeboscq, and B. Tsering-Xiao, On uniqueness for time harmonic anisotropic Maxwell's equations with piecewise regular coefficients, Math. Models Methods Appl. Sci. 22 (2012), 1250036.

[5] A. S. Bonnet-Ben Dhia, L. Chesnel, and P. Ciarlet, T-coercivity for scalar interface problems between dielectrics and metamaterials, ESAIM Math. Model. Numer. Anal. 46 (2012), 1363-1387.

[6] A. S. Bonnet-Ben Dhia, L. Chesnel, and P. Ciarlet, T-coercivity for the Maxwell problem with signchanging coefficients, Comm. Partial Differential Equations 39 (2014), 1007-1031.

[7] A. S. Bonnet-Ben Dhia, L. Chesnel, and X. Claeys, Radiation condition for a non-smooth interface between a dielectric and a metamaterial, Math. Models Methods Appl. Sci. 23 (2013) 1629-1662.

[8] D. Colton and R. Kress, Inverse acoustic and electromagnetic scattering theory, second ed., Applied Mathematical Sciences, vol. 98, Springer-Verlag, Berlin, 1998.

[9] M. Costabel and E. Stephan, A direct boundary integral equation method for transmission problems, J. Math. Anal. Appl. 106 (1985), 367-413.

[10] G. Csató, B. Dacorogna, and O. Kneuss, The pullback equation for differential forms. Progress in Nonlinear Differential Equations and their Applications, 83. Birkhäuser/Springer, New York, 2012.

[11] B. Dacorogna, Poincaré lemma in a star shaped domain, unpublished note, 2016.

[12] V. Girault, P.A. Raviart, Finite element methods for Navier-Stokes equations, theory and algorithms, Springer-Verlag, Berlin, 1986.

[13] H. Haddar, P. Joly, and H.-M. Nguyen, Generalized impedance boundary conditions for scattering problems from strongly absorbing obstacles: the case of Maxwell's equations, Math. Models Methods Appl. Sci. 18 (2008), 1787-1827.

[14] H. Kang, M. Lim, and S. Yu, Spectral resolution of the Neumann-Poincaré operator on intersecting disks and analysis of plasmon resonance, Arch. Ration. Mech. Anal.226 (2017), 83-115.

[15] R. V. Kohn, J. Lu, B. Schweizer, and M. I. Weinstein, A variational perspective on cloaking by anomalous localized resonance, Comm. Math. Phys. 328 (2014), 1-27.

[16] R. Leis, Initial-boundary value problems in mathematical physics, B. G. Teubner, Stuttgart; John Wiley \& Sons, Ltd., Chichester, 1986.

[17] G. W. Milton and N-A. P. Nicorovici, On the cloaking effects associated with anomalous localized resonance, Proc. R. Soc. Lond. Ser. A 462 (2006), 3027-3059. 
[18] P. Monk. Finite element methods for Maxwell's equations. Numerical Mathematics and Scientific Computation. Oxford University Press, New York, 2003.

[19] H-M. Nguyen, Asymptotic behavior of solutions to the Helmholtz equations with sign changing coefficients, Trans. Amer. Math. Soc. 367 (2015), 6581-6595.

[20] H.-M. Nguyen, Superlensing using complementary media, Ann. Inst. H. Poincaré Anal. Non Linéaire 32 (2015), 471-484.

[21] H.-M. Nguyen, Cloaking via anomalous localized resonance for doubly complementary media in the quasistatic regime, J. Eur. Math. Soc. (JEMS) 17 (2015), 1327-1365.

[22] H.-M. Nguyen, Cloaking using complementary media in the quasistatic regime, Ann. Inst. H. Poincaré Anal. Non Linéaire, 33 (2016), 1509-1518.

[23] H.-M. Nguyen, Limiting absorption principle and well-posedness for the Helmholtz equation with sign changing coefficients, J. Math. Pures Appl. 106 (2016), 342-374.

[24] H.-M. Nguyen, Cloaking an arbitrary object via anomalous localized resonance: the cloak is independent of the object, SIAM J. Math. Anal. 49 (2017), 3208-3232.

[25] H.-M. Nguyen, Superlensing using complementary media and reflecting complementary media for electromagnetic waves, Adv. Nonlinear Anal. 7 (2018), 449-467.

[26] H.-M. Nguyen, Cloaking using complementary media for electromagnetic waves, ESAIM Control Optim. Calc. Var. 25 (2019) 29.

[27] H.-M. Nguyen, Cloaking via anomalous localized resonance for doubly complementary media in the finite frequency regime, J. Anal. Math., to appear, https://doi.org/10.1007/s11854-019-0024-8.

[28] H.-M. Nguyen, The invisibility via anomalous localized resonance of a source for electromagnetic waves, Res. Math. Sci, revised, https://arxiv.org/abs/1906.06152.

[29] H.-M. Nguyen and H. L. Nguyen, Cloaking using complementary media for the Helmholtz equation and a three spheres inequality for second order elliptic equations, Trans. Amer. Math. Soc. Ser. B 2 (2015), 93-112.

[30] H.-M. Nguyen and Q.-H. Nguyen, Discreteness of interior transmission eigenvalues revisited. Calc. Var. Partial Differential Equations , 56 (2017), Art. 51, 38.

[31] T. Nguyen and J.-N. Wang, Quantitative uniqueness estimate for the Maxwell system with Lipschitz anisotropic media, Proc. Amer. Math. Soc. 140 (2012), 595-605.

[32] N. A. Nicorovici, R. C. McPhedran, and G. M. Milton, Optical and dielectric properties of partially resonant composites, Phys. Rev. B 49 (1994), 8479-8482.

[33] P. Ola, Remarks on a transmission problem, J. Math. Anal. Appl. 196 (1995), 639-658.

[34] R. A. Shelby, D. R. Smith, and S. Schultz, Experimental Verification of a Negative Index of Refraction, Science 292 (2001), 77-79.

[35] M. H. Protter, Unique continuation for elliptic equations, Trans. Amer. Math. Soc. 95 (1960), 81-91.

[36] V. G. Veselago, The electrodynamics of substances with simultaneously negative values of $\varepsilon$ and $\mu$, Usp. Fiz. Nauk 92 (1964), 517-526.

(H.-M. Nguyen) Department of Mathematics, EPFL SB CAMA, Station 8, CH-1015 LAUSANNE, SwitzerLand.

E-mail address: hoai-minh.nguyen@epfl.ch

(S. Sil) Forschungsinstitut für Mathematik, ETH Zurich

Rämistrasse 101, 8092 Zurich, Switzerland.

E-mail address: swarnendu.sil@fim.math.ethz.ch 